\title{
4-Aryl-1,3,2-Oxathiazolylium-5-olates as pH-Controlled NO-Donors: The Next Generation of $S$-Nitrosothiols
}

Dongning Lu, ${ }^{a}$ Janos Nadas, ${ }^{a}$ Guisheng Zhang, ${ }^{c}$ Wesley Johnson, ${ }^{b}$ Jay L. Zweier, ${ }^{b}$ Arturo J. Cardounel, ${ }^{b}$ Frederick A. Villamena, ${ }^{b^{*}}$ and Peng George Wang ${ }^{a^{*}}$

${ }^{\mathrm{a}}$ Departments of Biochemistry and Chemistry; ${ }^{\mathrm{b}}$ The Davis Heart and Lung Research Institute, and the Division of Cardiovascular Medicine, Department of Internal Medicine, College of Medicine; The Ohio State University; ${ }^{\mathrm{c} C o l l e g e}$ of Chemistry and Environmental Science, Henan Normal University, Henan Xinxiang 453007, P. R.

\section{China}

*Correspondence to: Peng George Wang, Phone: (614) 292-9884. Fax: (614) 688-3106. E-mail:

wang.892@osu.edu. Frederick A. Villamena, Phone: (614) 292-8215. Fax: (614) 292-8454. E-mail: frederick.villamena@osumc.edu

\section{Supporting Information}

\section{Contents}

NMR Spectra

S2-9

Stability Studies on UV-Vis

S10-11

EPR Spectra

Mass Spectra

Cyclic Voltammetry Spectra

Computional Results

S20-23

Complete ref (83) 

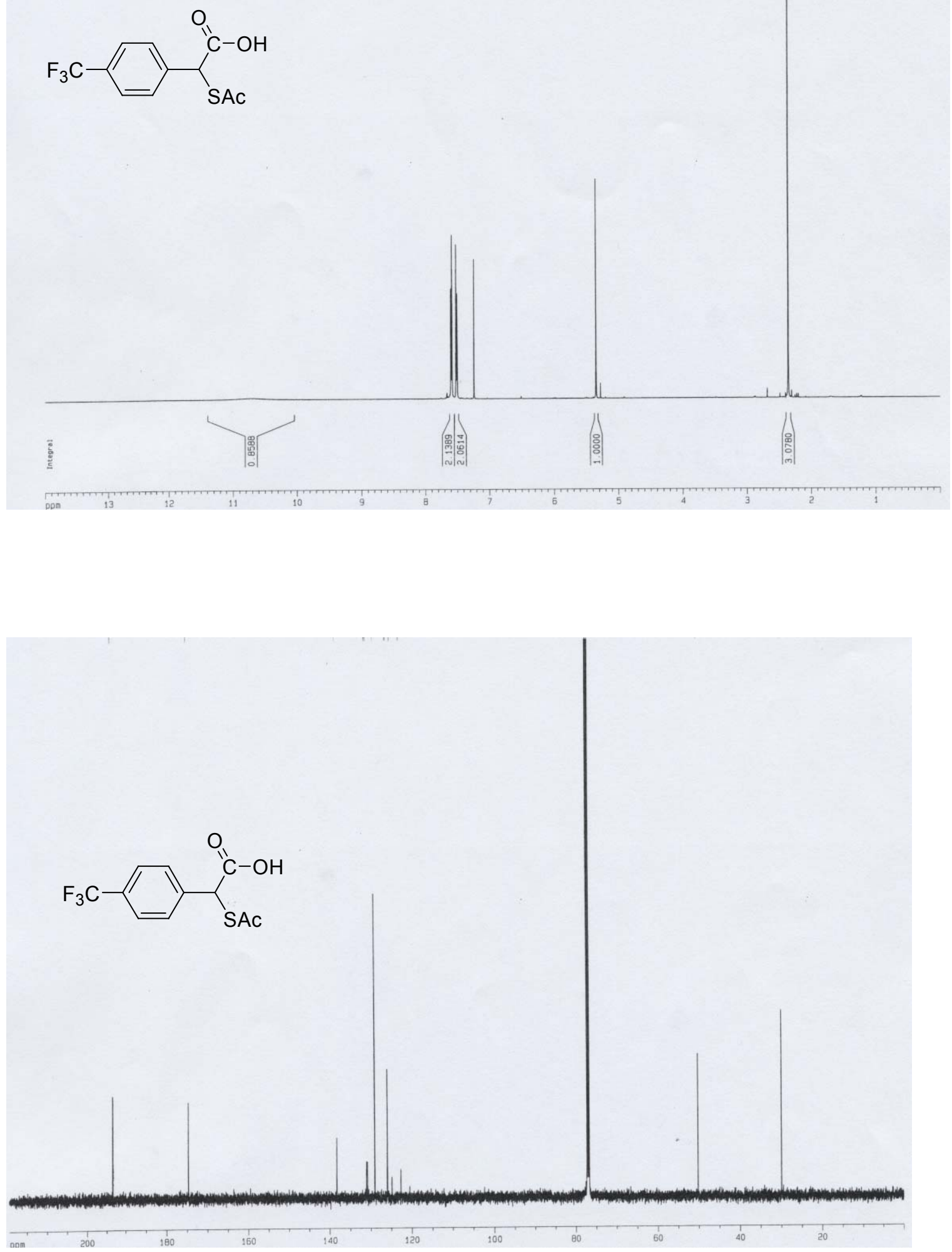


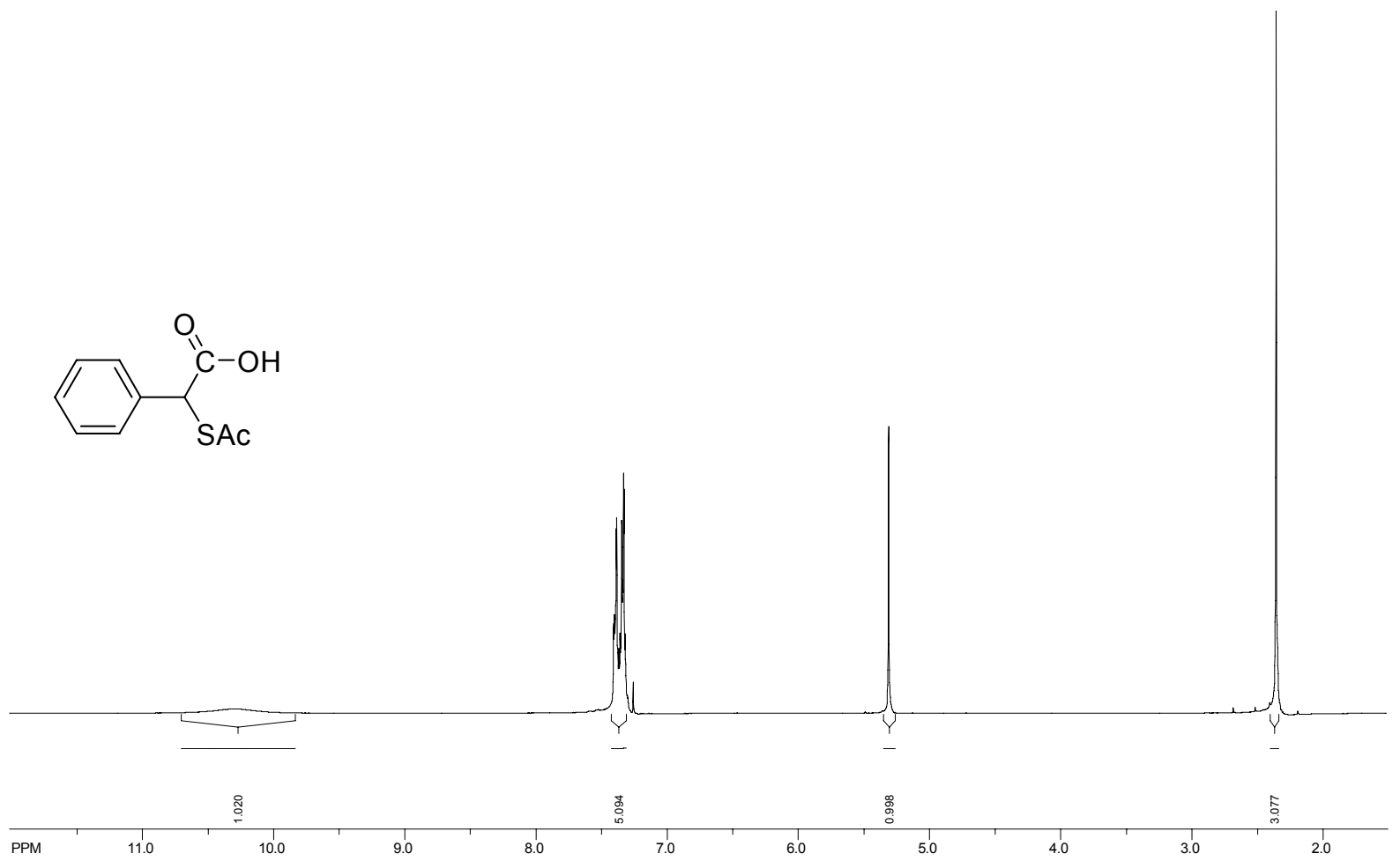

SpinWorks 2.3: 2b in CDCl3 at rt on $400 \mathrm{M}$

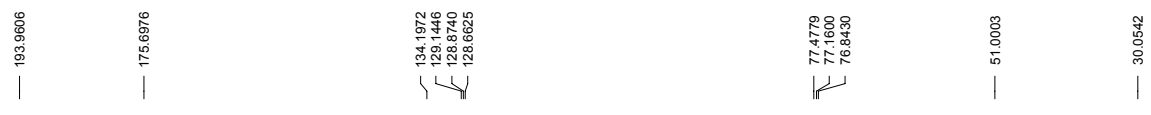

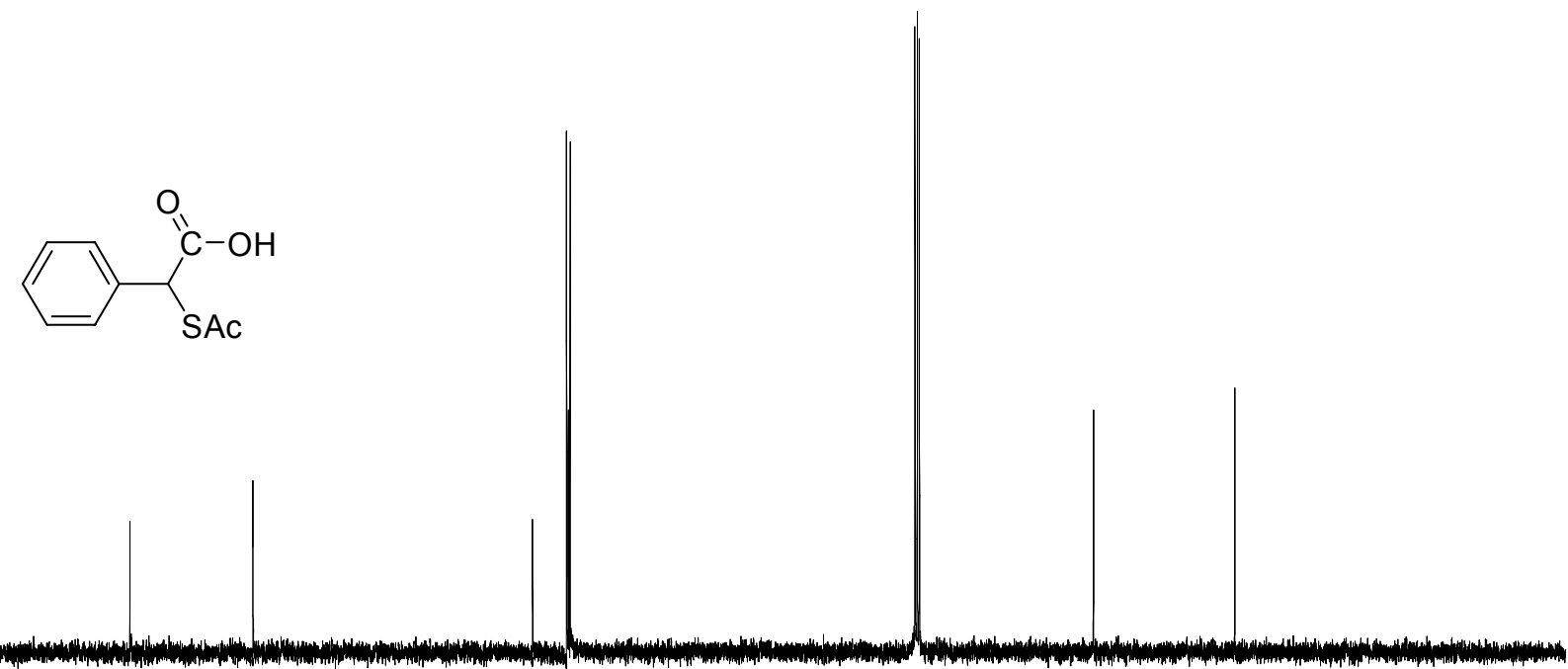



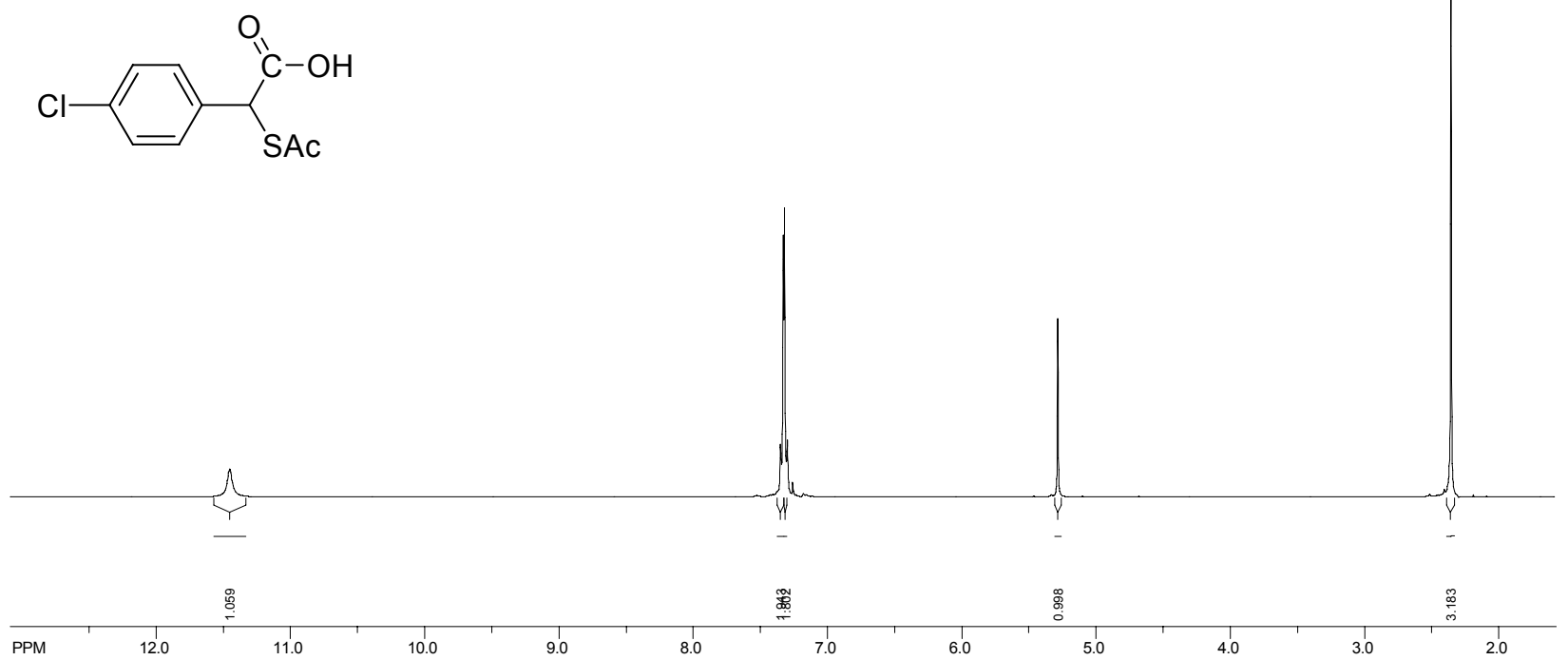

SpinWorks 2.3:

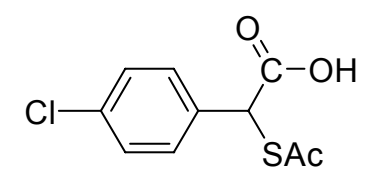

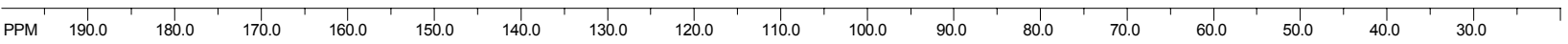




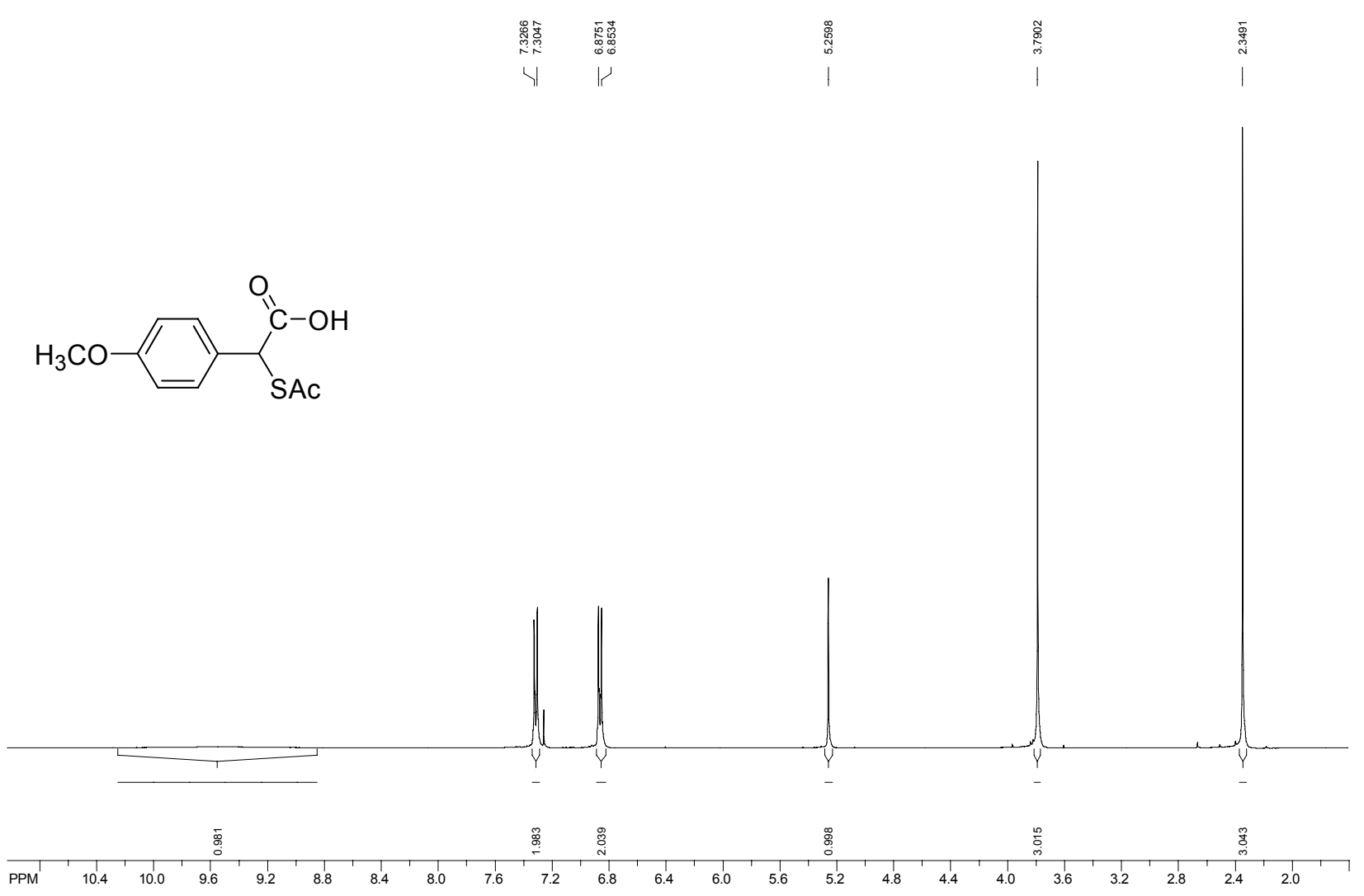

SpinWorks 2.3: $2 \mathrm{~b}$ in $\mathrm{CHCl} 3$ at rt on $400 \mathrm{M}$

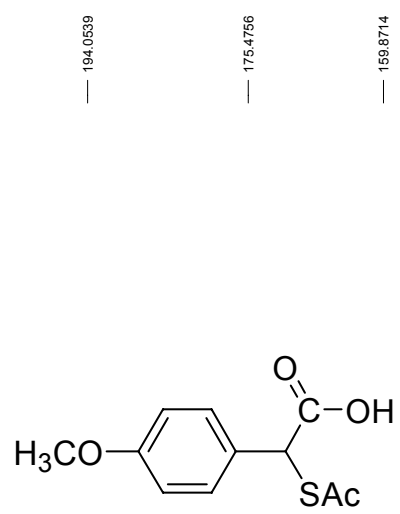

|
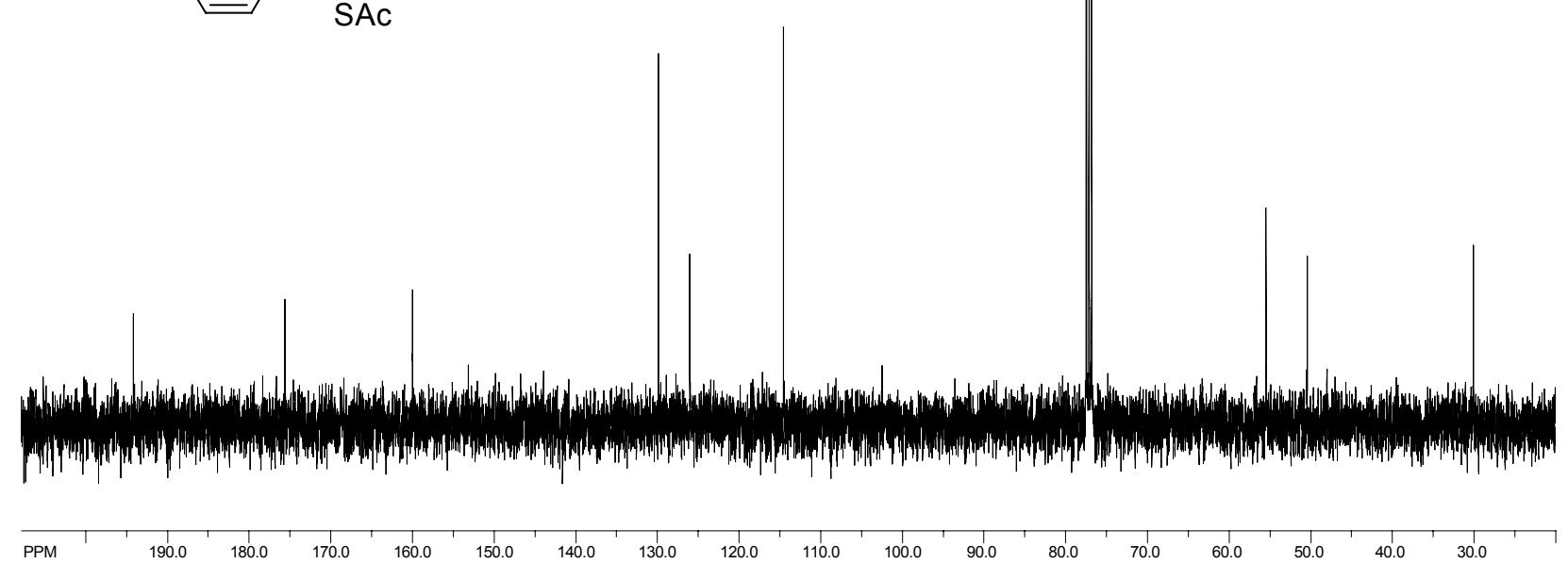
SpinWorks 2.3: 3a in DMSO

墖重

$4 \quad$ r

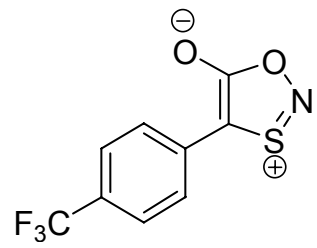
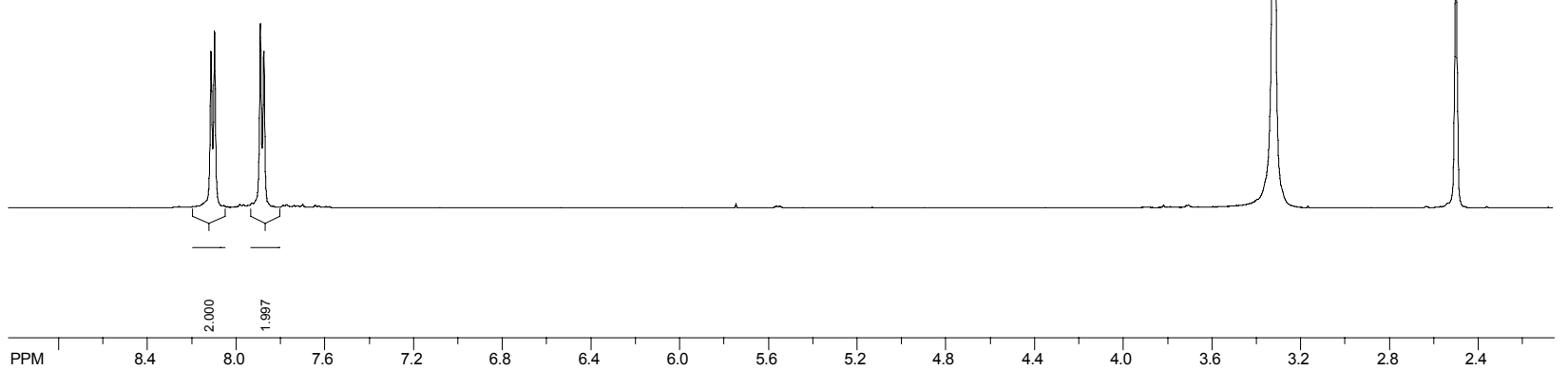

SpinWorks 2.3: 3a in $\mathrm{CHCl} 3$

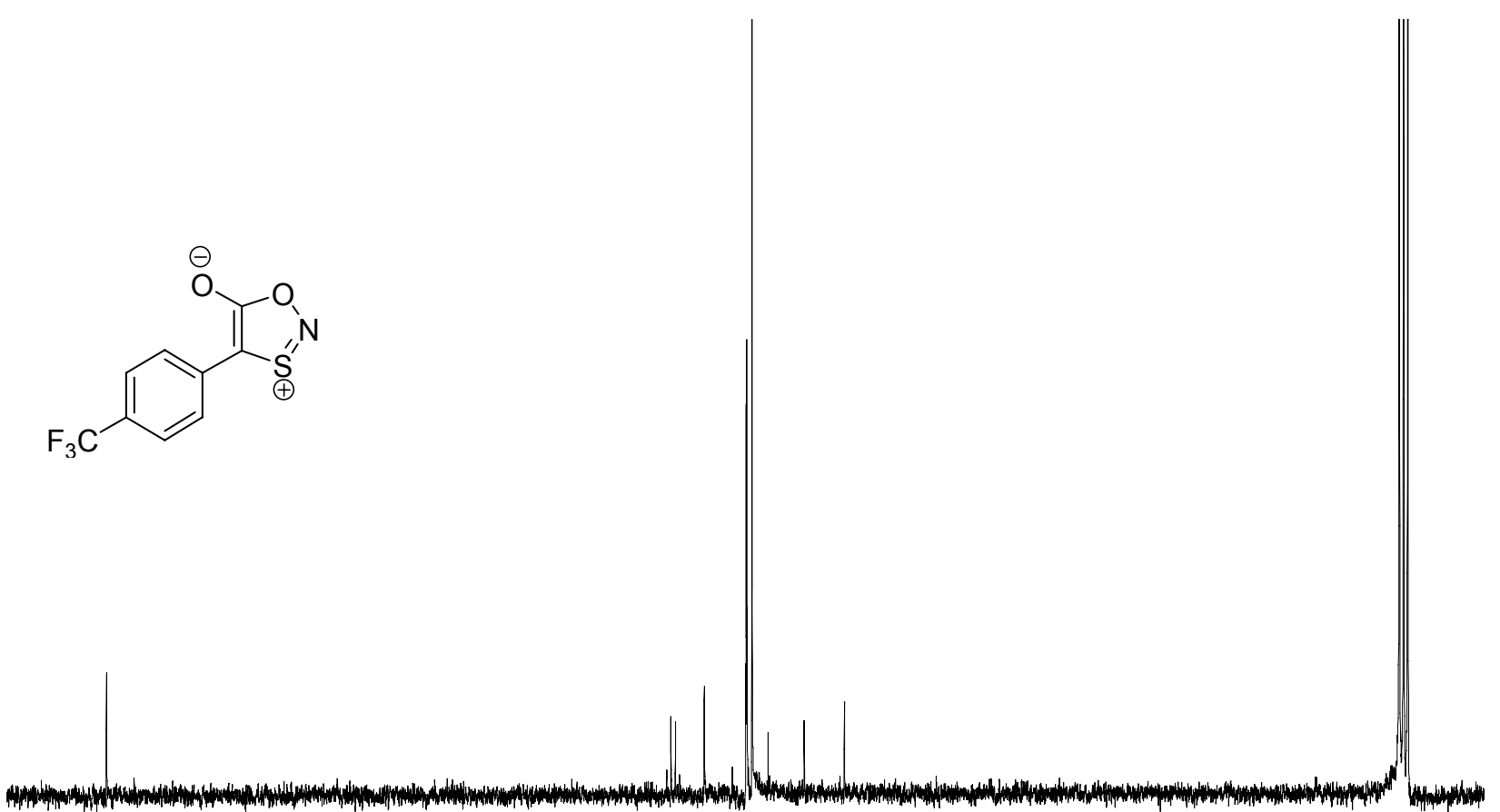

PPM

170.0

160.0

150.0

140.0

130.0

120.0

110.0

100.0

90.0

80.0 
SpinWorks 2.3: Lu's sample2 in DMSO-d6 at rt on $500 \mathrm{M}$
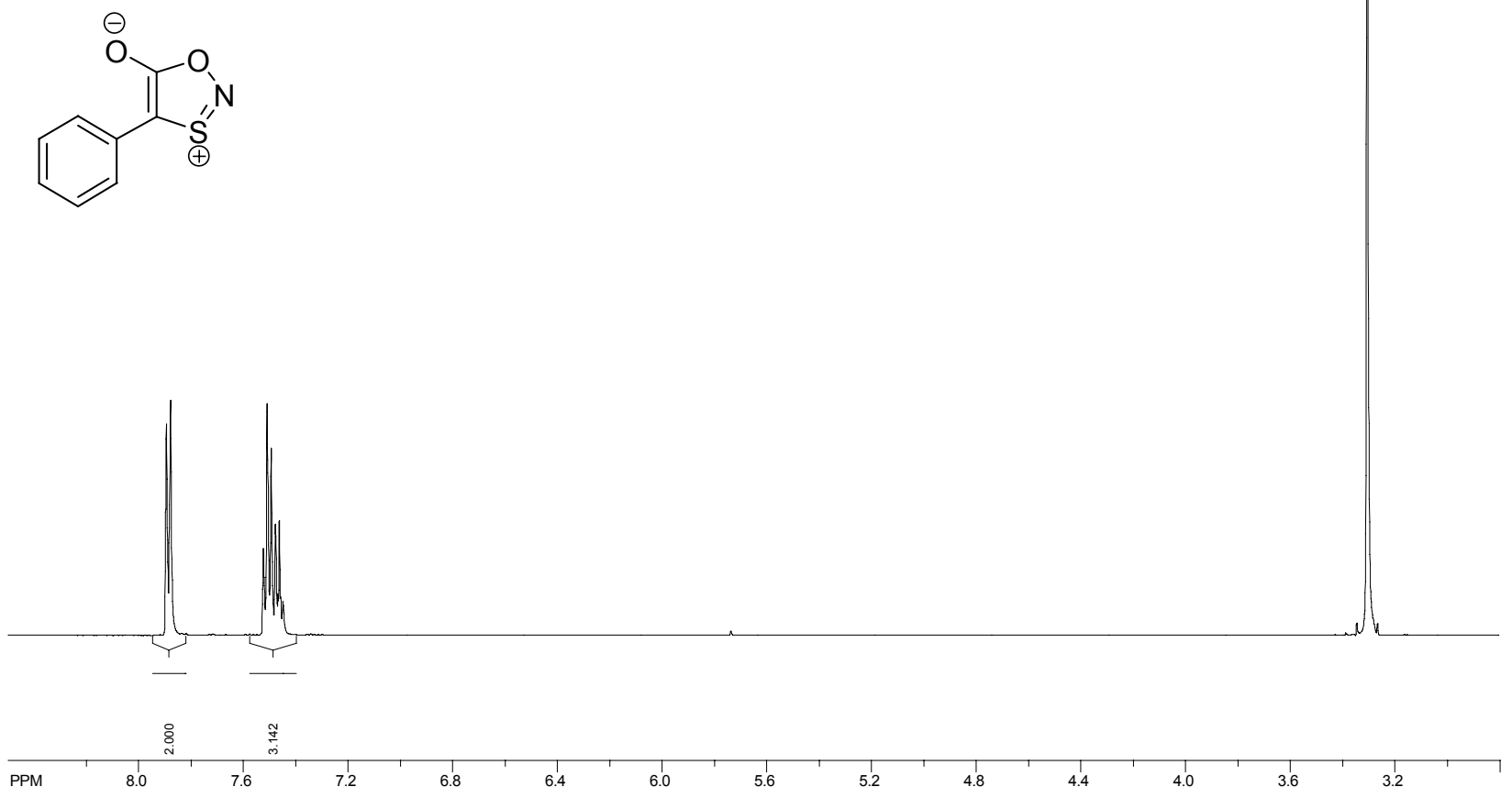

SpinWorks 2.3: Lu's sample in DMSO-d6 at it on $500 \mathrm{M}$
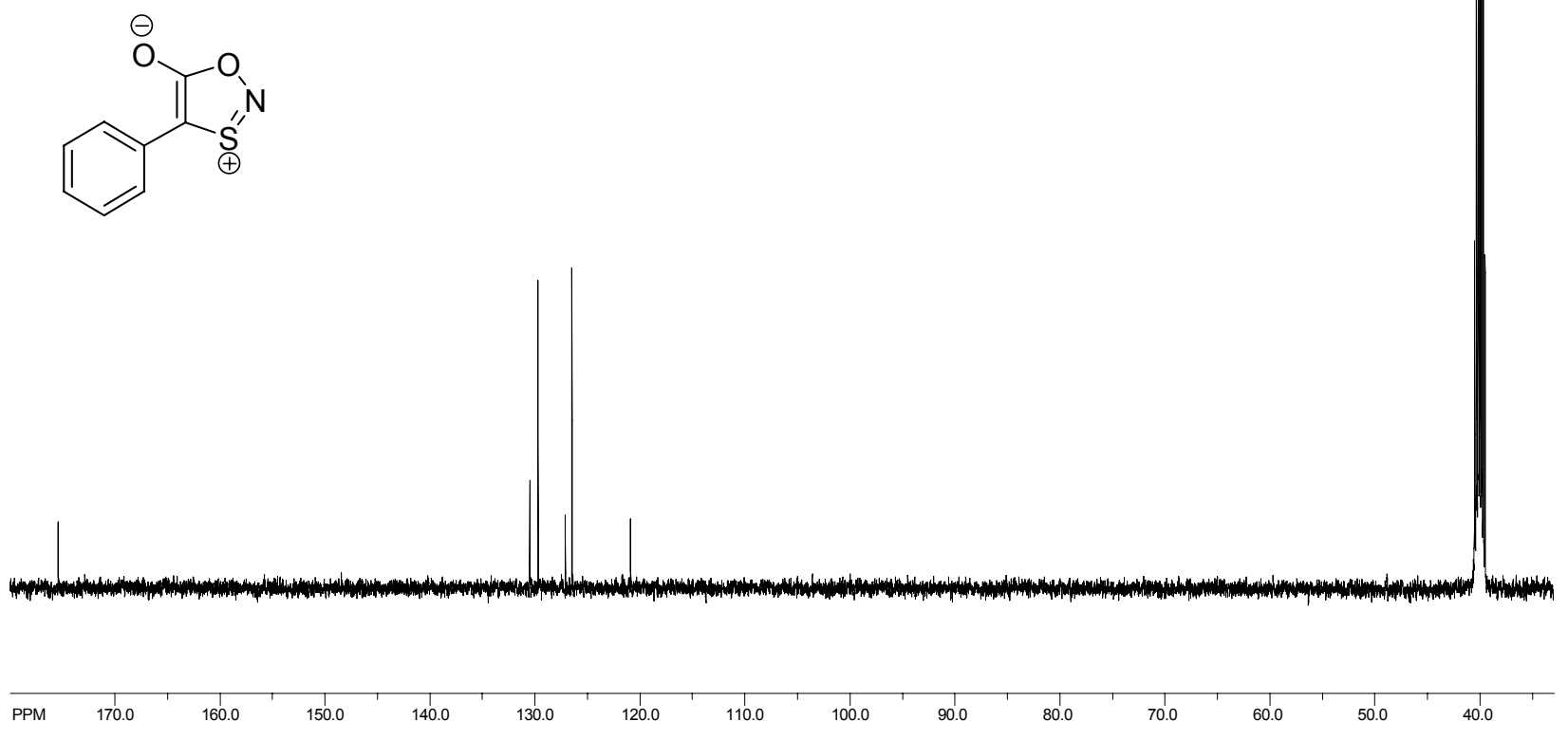

S7 
SpinWorks 2.3: 3c in DMSO at it on $400 \mathrm{M}$

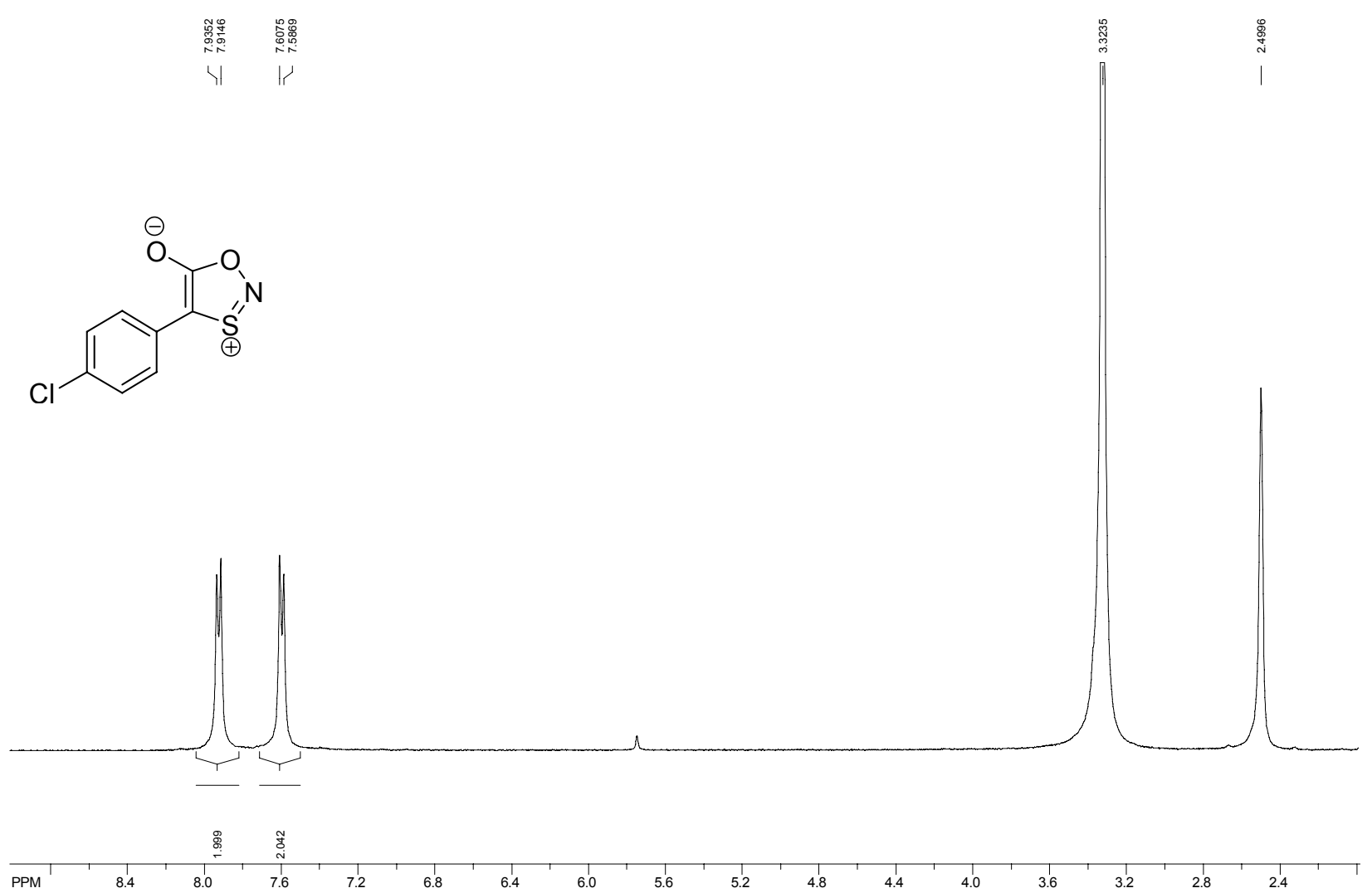

SpinWorks 2.3: 3c in DMSO at rt on $400 \mathrm{M}$<smiles></smiles>

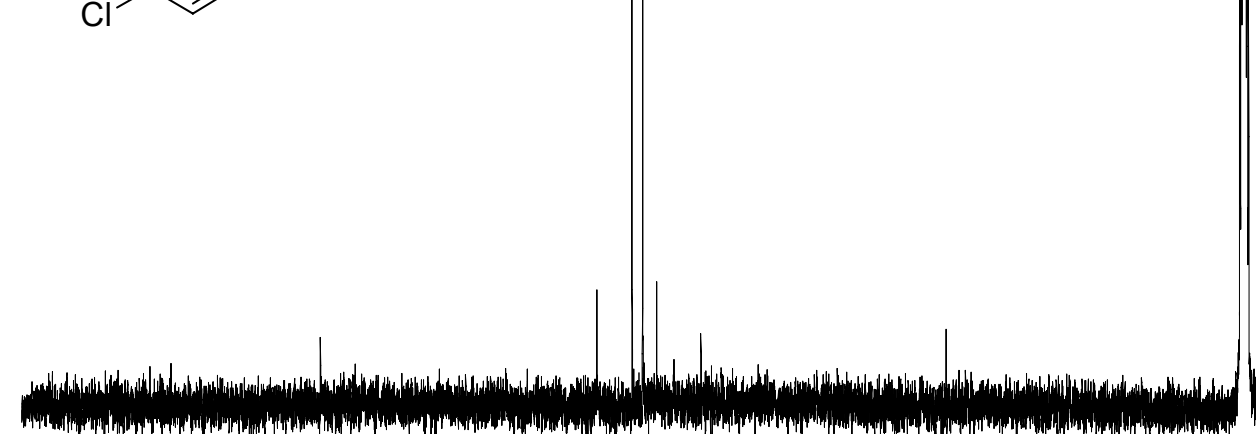

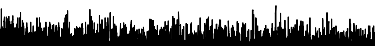

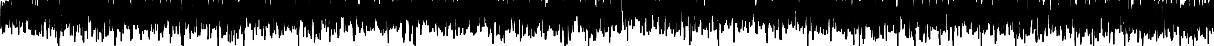

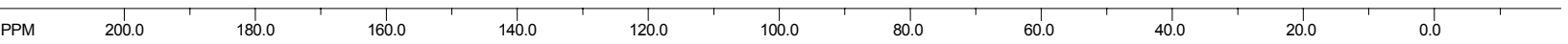



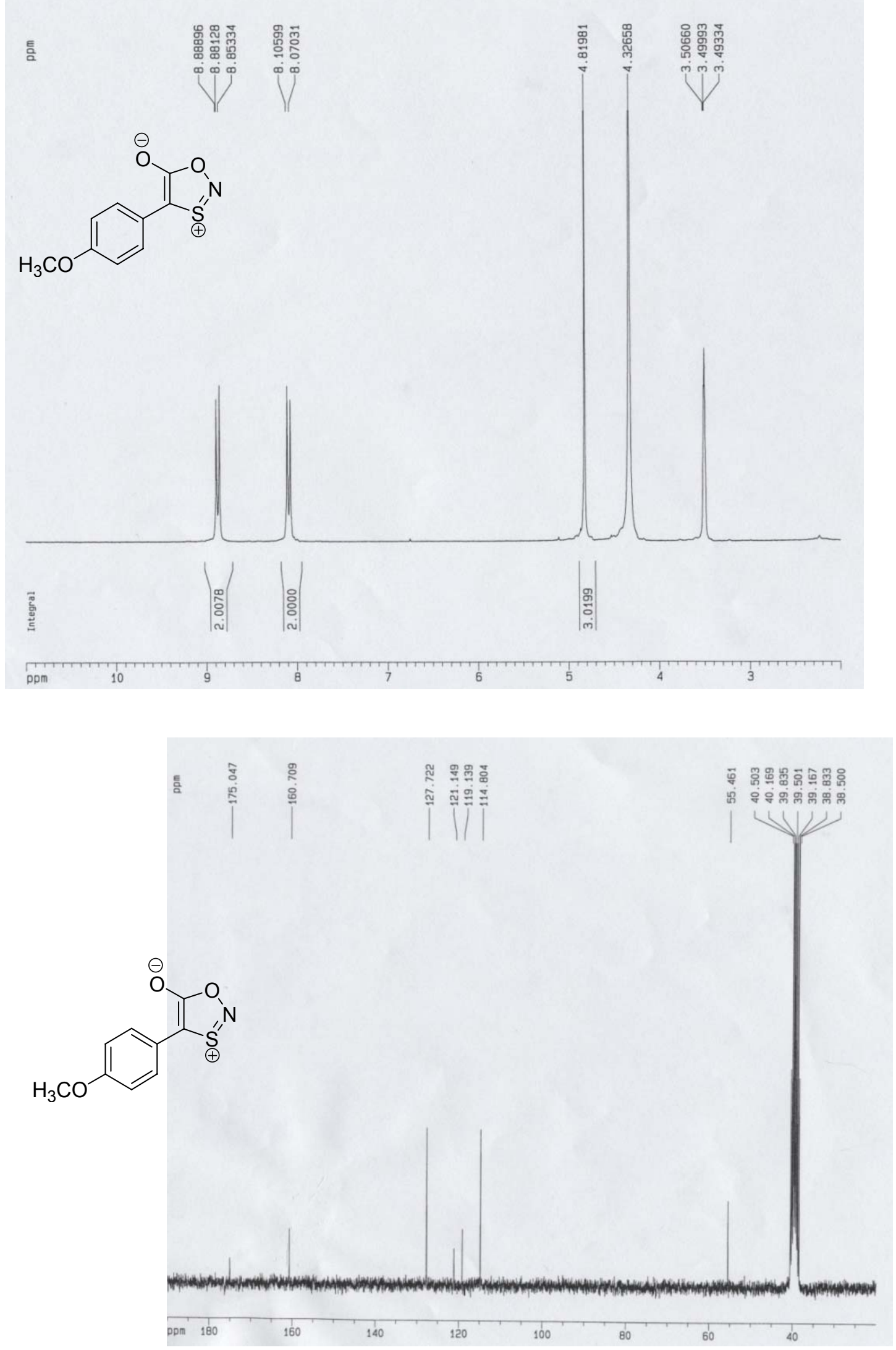

Figure S1. NMR Spectra of 3a-3d. 

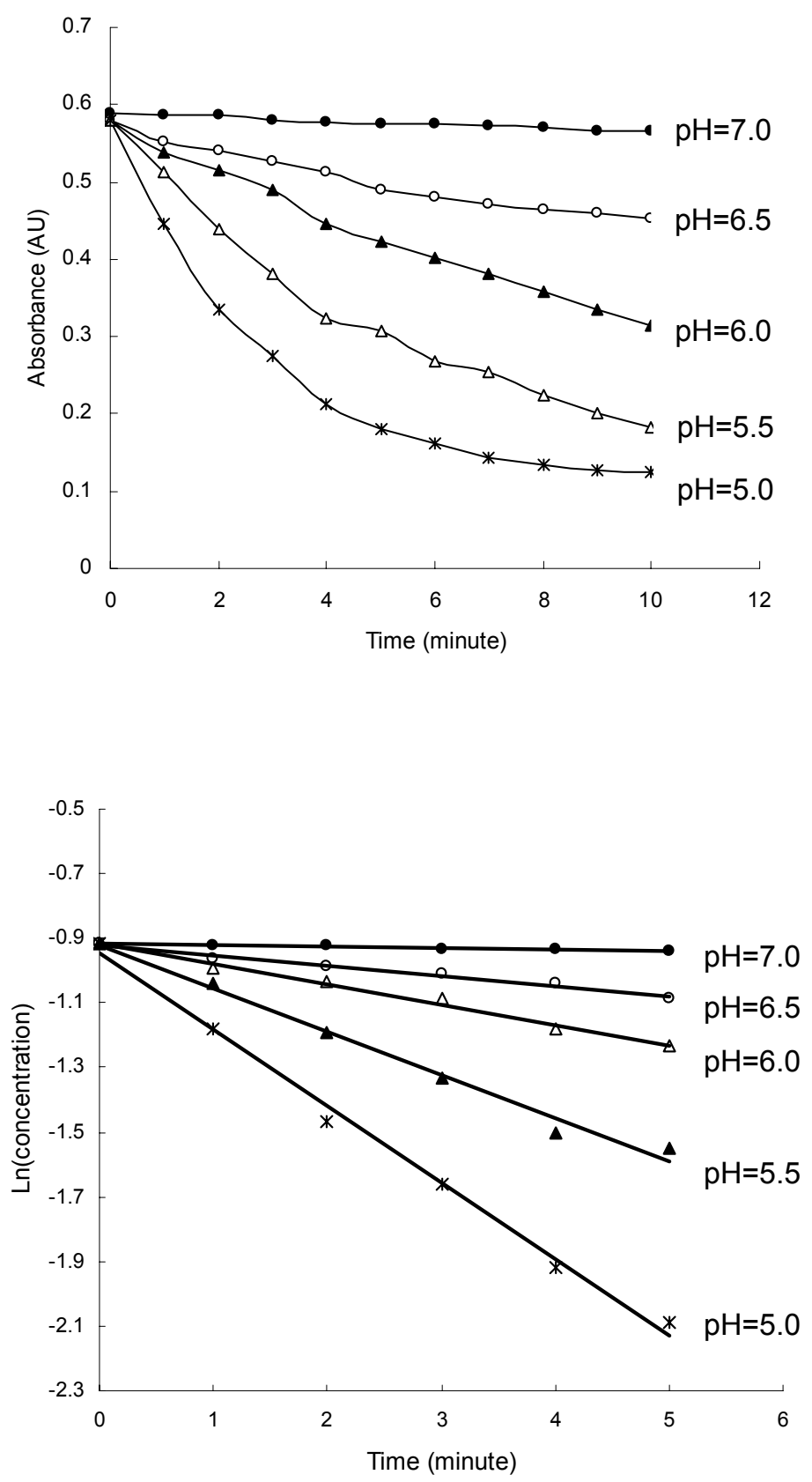

Figure S2. Decomposition plot and $\ln$ [concentration] vs. Time plot of $3 \mathbf{b}(0.4 \mathrm{mM})$ in $0.1 \mathrm{M}$ phosphate at $\mathrm{pH}$ 5.0 7.0. The $\mathrm{Ln}$ (concentration) vs. time plot was obtained in the first five minutes, the slope of the regression line is the decomposition rate constant $k$ for persodo-first-order reaction at a certain $\mathrm{pH}$. 

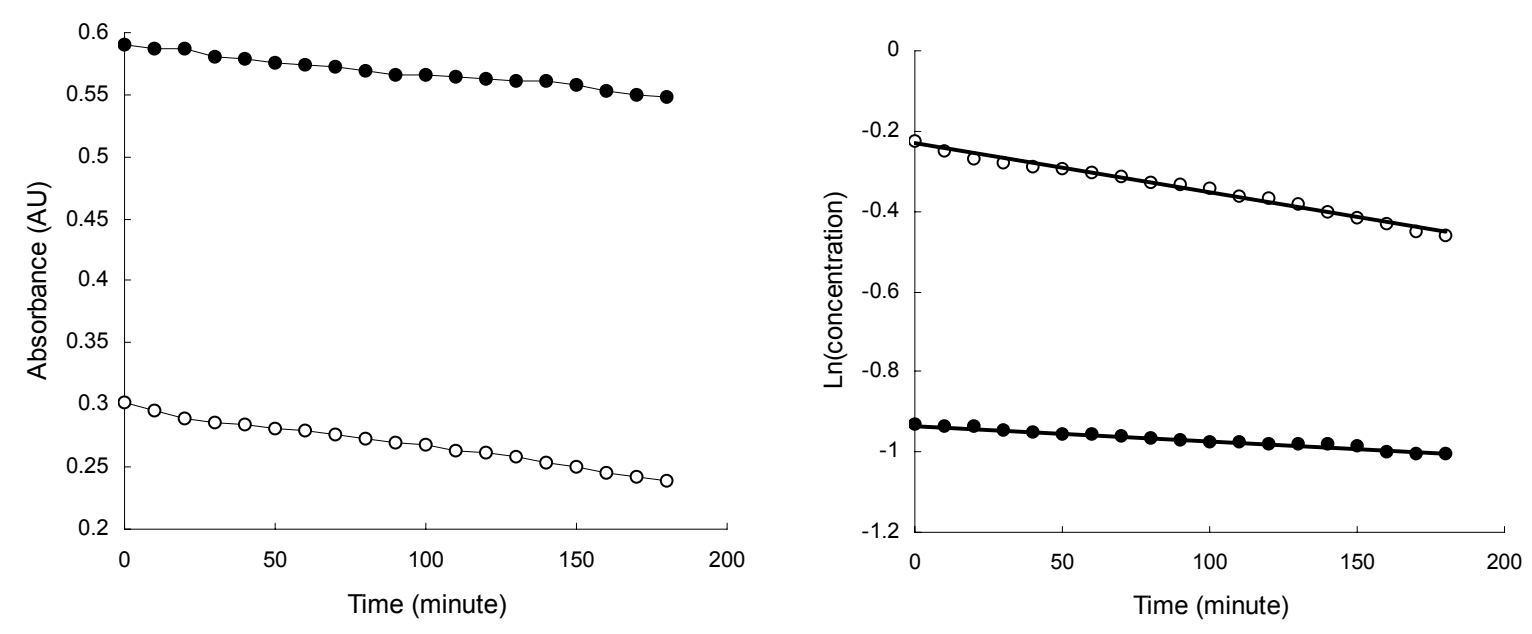

Figure S3. Decomposition plot and $\ln [$ concentration] vs. Time plot of $\mathbf{3 b}(\bullet, 0.4 \mathrm{mM})$ and GSNO (०, $0.8 \mathrm{mM}$ ) in $0.1 \mathrm{M}$ phosphate buffer in the presence of stray light, $\mathrm{pH} 7.0$. UV was measured at $587 \mathrm{~nm}$ (3b) and $335 \mathrm{~nm}$ (GSNO).
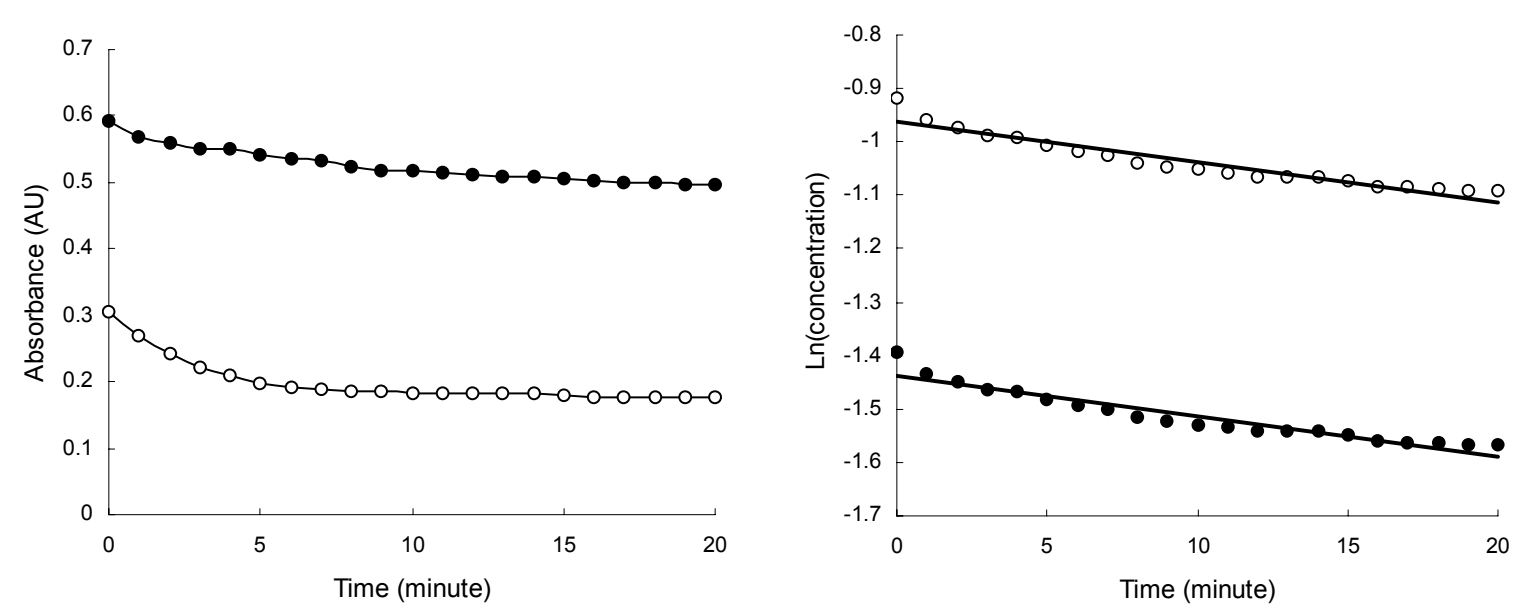

Figure S4. Decomposition plot and $\ln [$ concentration] vs. Time plot of $\mathbf{3 b}(\bullet, 0.4 \mathrm{mM})$ and GSNO (०, $0.8 \mathrm{mM})$ in $0.1 \mathrm{M}$ phosphate buffer in the presence of $\mathrm{Fe}^{2+}(10 \mu \mathrm{M}, 20 \mu \mathrm{M}$, respectively), $\mathrm{pH} 7.0$. UV was measured at $587 \mathrm{~nm}(3 \mathbf{b})$ and $335 \mathrm{~nm}(\mathrm{GSNO})$. 
EPR spin trapping using ${ }^{17} \mathrm{O}$-enriched $\mathrm{H}_{2}{ }^{17} \mathrm{O}$ was carried out to further rule out the possibility of a DMPO-OH formation via $\mathrm{H}$-atom abstraction from $\mathrm{H}_{2} \mathrm{O}$ by the thiyl radical 6 to form the thiol and $\mathrm{HO}^{\circ}$ radical. Results show no formation of DMPO- ${ }^{17} \mathrm{OH}$ adduct and that only the DMPO-SR adduct was formed from $50 \mathrm{mM}$ DMPO, $10 \mathrm{mM}$ 3a, in $(10 \% / 90 \%) \mathrm{H}_{2}{ }^{17} \mathrm{O} / \mathrm{H}_{2}{ }^{16} \mathrm{O}$ at $\mathrm{pH} 5$ (Figure $\mathrm{S} 5 \mathrm{c}$ of the Supporting Information). To confirm this observation, the adduct, DMPO- ${ }^{17} \mathrm{OH}$, was independently generated and detected by EPR based on the $\mathrm{Fe}^{3+}$ catalyzed nucleophilic addition of $\mathrm{H}_{2}{ }^{17} \mathrm{O}$ to nitrone and subsequent oxidation to DMPO- ${ }^{17} \mathrm{OH}$ adduct ${ }^{1}$ with $a_{\mathrm{N}}=14.98 \mathrm{G}, a_{\beta-\mathrm{H}}=14.72 \mathrm{G}, a_{\mathrm{O}-17}=4.65 \mathrm{G}$. (Figure S5b of the Supporting Information). The characteristic EPR signal for DMPO- ${ }^{17} \mathrm{OH}$ was further confirmed by UV photolysis of $50 \mathrm{mM}$ DMPO and $100 \mu \mathrm{M}(80 \% / 20 \%) \mathrm{H}_{2}{ }^{17} \mathrm{O}_{2} / \mathrm{H}_{2}{ }^{16} \mathrm{O}_{2}$ (Figure S5a of the Supporting Information). Computational studies also show that $\mathrm{H}$-atom abstraction from $\mathrm{H}_{2} \mathrm{O}$ by the thiyl radical to form $\mathrm{HO}^{\circ}$ is highly unfavorable with free energies of reaction of $\Delta G_{\mathrm{rxn}, \mathrm{aq}}=28.8-32.9$ $\mathrm{kcal} / \mathrm{mol}$ at the PCM/B3LYP/6-31+G**//B3LYP/6-31G* level of theory.

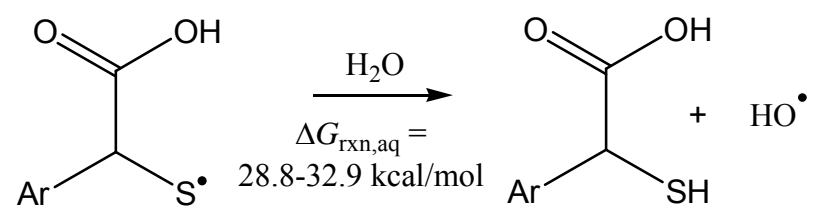

(1) Hanna, P. M.; Chamulitrat, W.; Mason, R. P. Arch. Biochem. Biophys. 1992, 296, 640. 

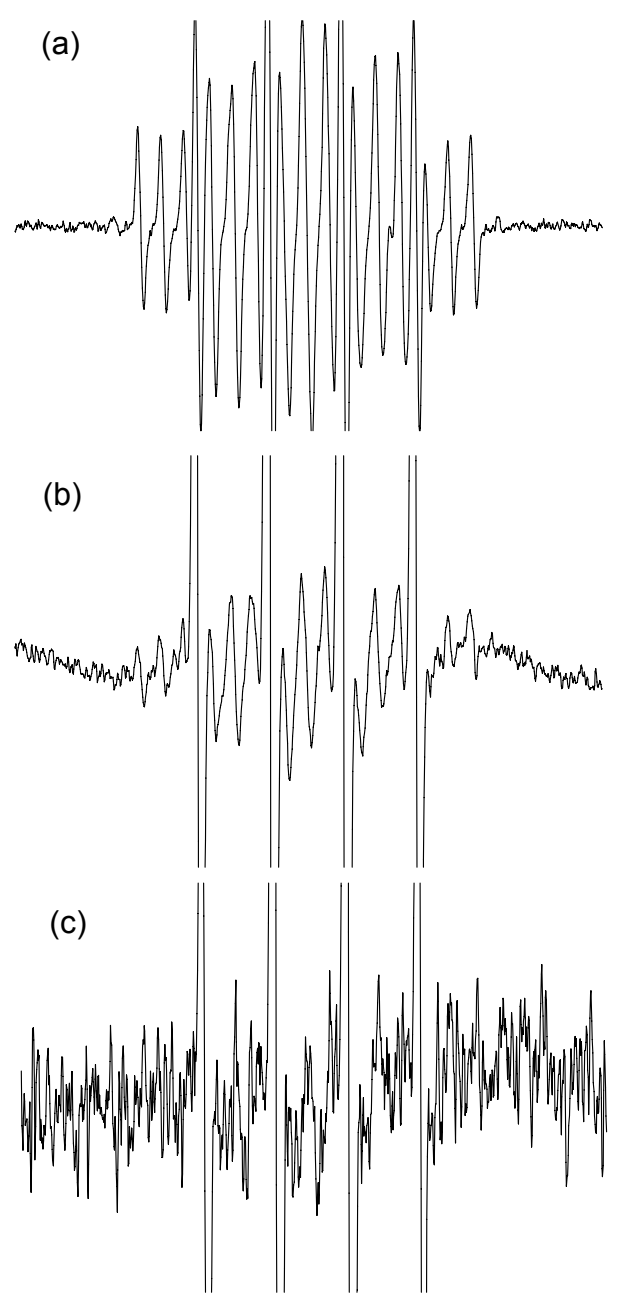

Figure S5. X-band EPR spectra of (a) UV photolysis of $50 \mathrm{mM}$ DMPO and $100 \mu \mathrm{M}(80 \% / 20 \%)$ $\mathrm{H}_{2}{ }^{17} \mathrm{O}_{2} / \mathrm{H}_{2}{ }^{16} \mathrm{O}_{2}$ showing the formation of DMPO- ${ }^{17} \mathrm{OH}\left(a_{\mathrm{N}}=14.98 \mathrm{G}, a_{\beta-\mathrm{H}}=14.72 \mathrm{G}, a_{\mathrm{O}-17}=4.65 \mathrm{G}\right)$ and DMPO- ${ }^{16} \mathrm{OH}$ (as overshoot, $a_{\mathrm{N}}=15.00 \mathrm{G}, a_{\beta-\mathrm{H}}=14.75 \mathrm{G}$ ) adducts; (b) DMPO- ${ }^{17} \mathrm{OH}$ and DMPO${ }^{16} \mathrm{OH}$ from $50 \mathrm{mM}$ DMPO and $\sim 100 \mu \mathrm{M} \mathrm{FeCl}_{3}$ in $(10 \% / 90 \%) \mathrm{H}_{2}{ }^{17} \mathrm{O} / \mathrm{H}_{2}{ }^{16} \mathrm{O}$; (c) DMPO-SR adduct from $50 \mathrm{mM}$ DMPO, $10 \mathrm{mM}$ 3a, in $(10 \% / 90 \%) \mathrm{H}_{2}{ }^{17} \mathrm{O} / \mathrm{H}_{2}{ }^{16} \mathrm{O}$ at $\mathrm{pH} 5$. 

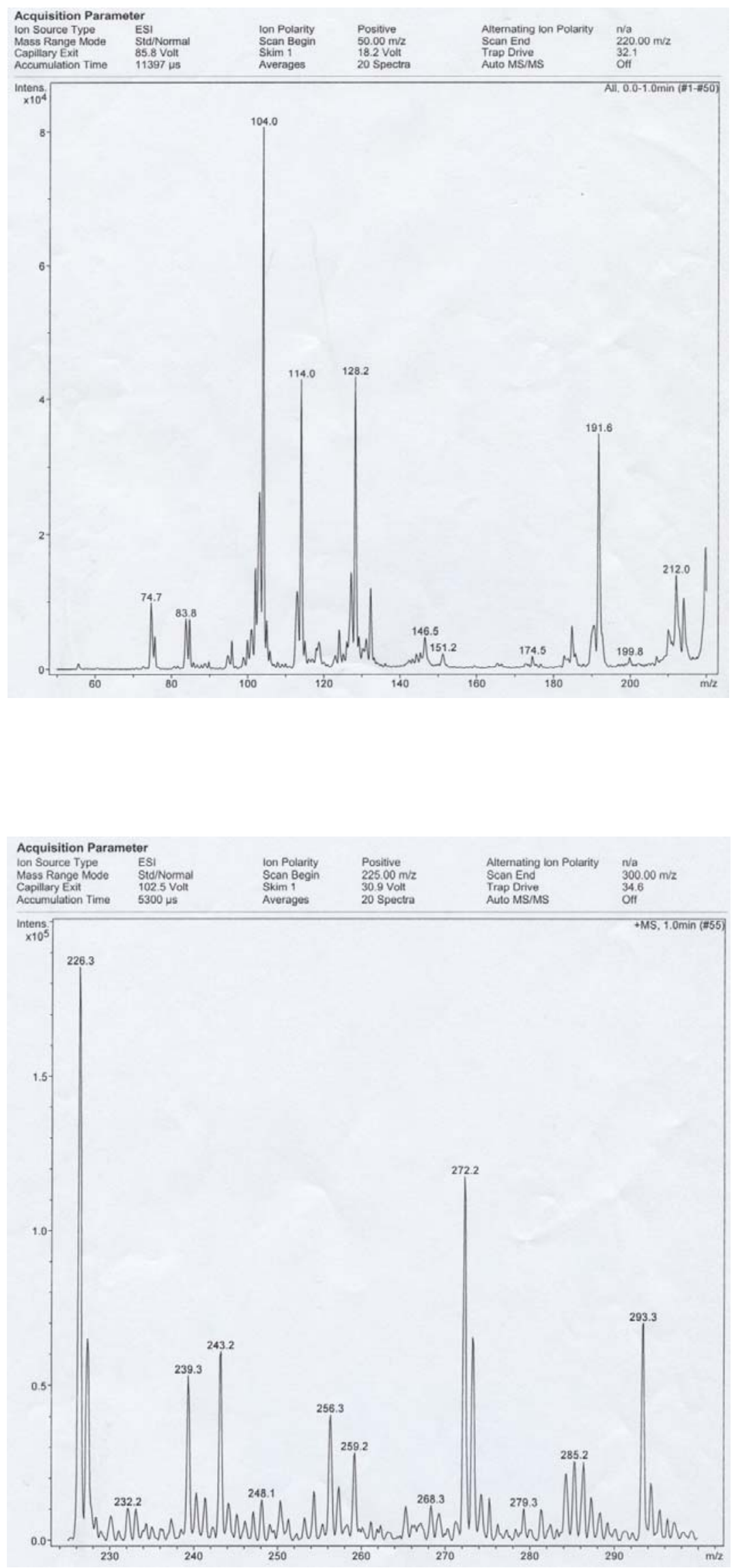

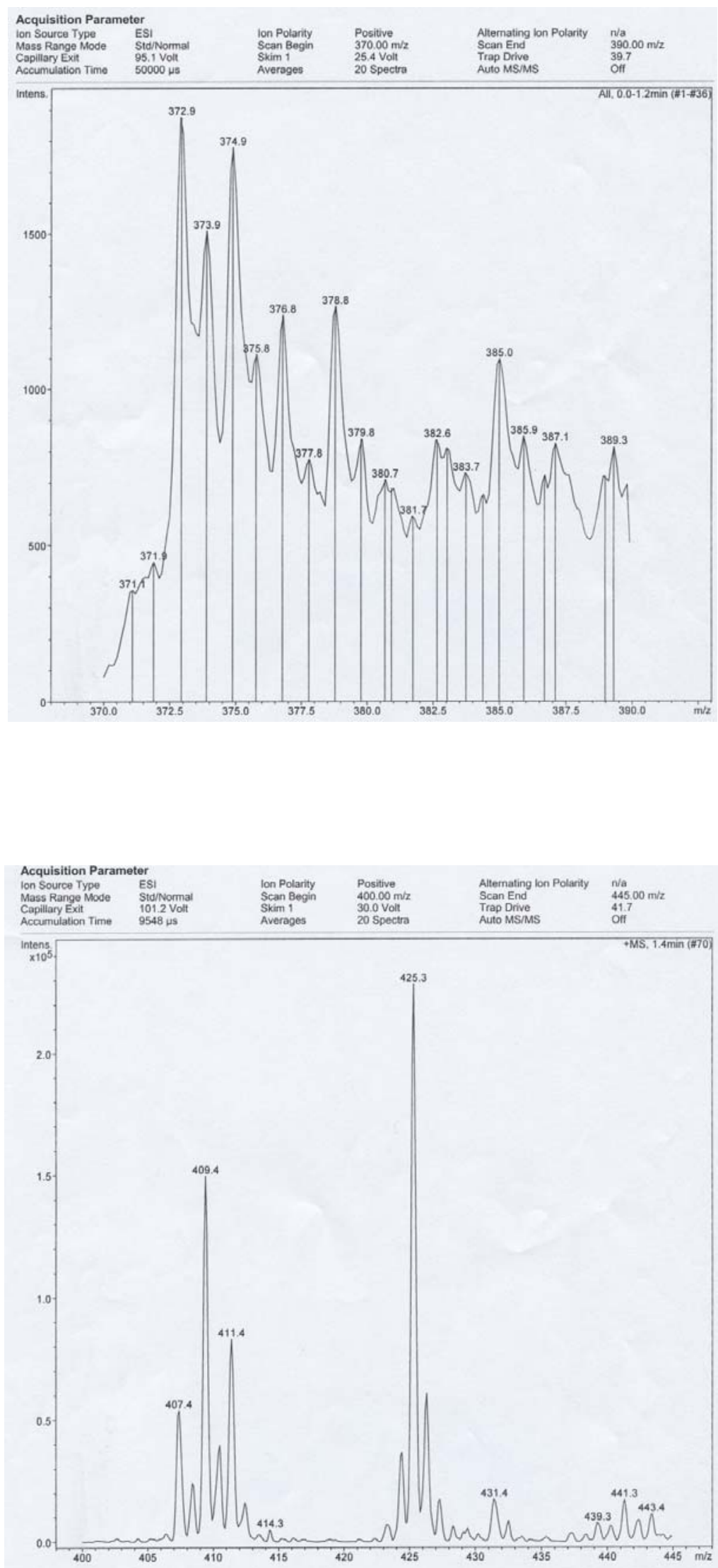

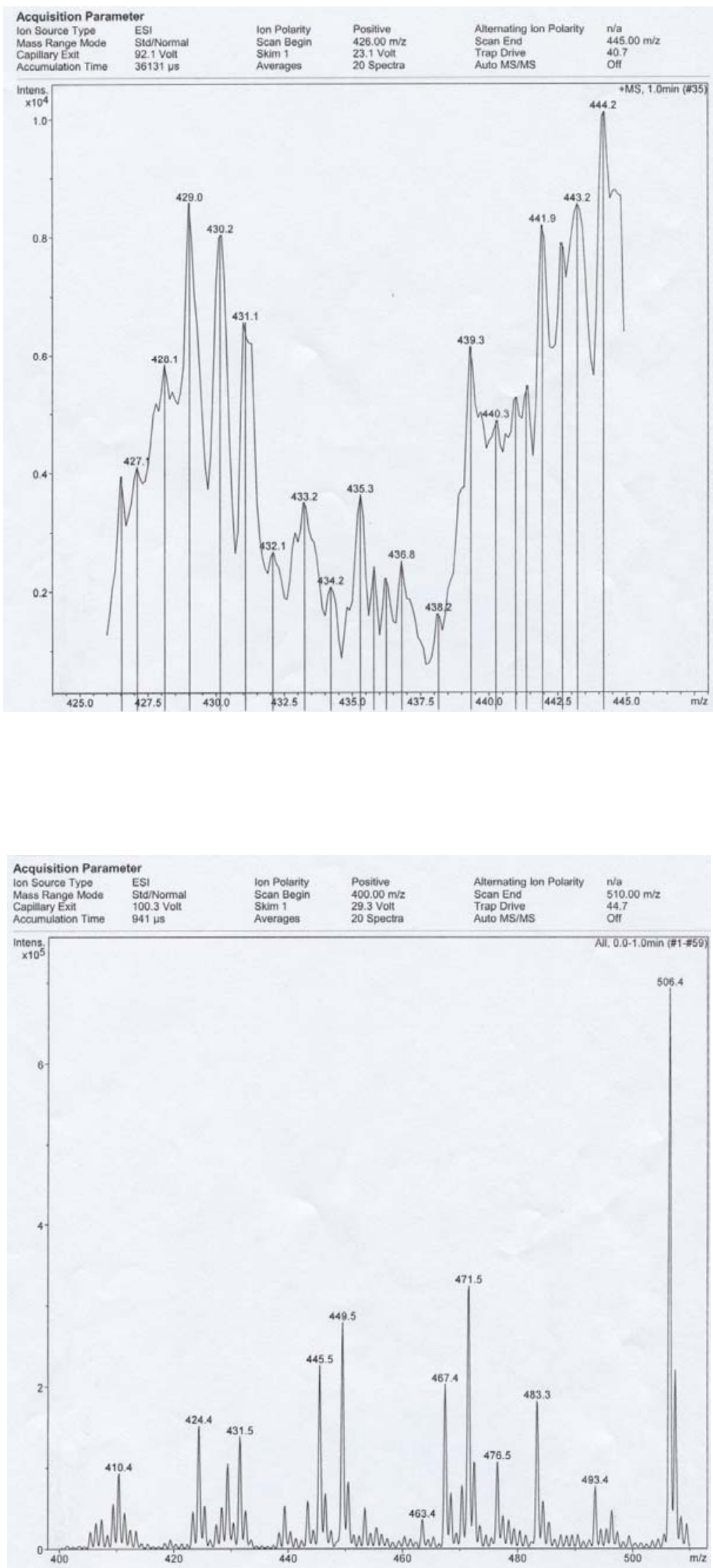


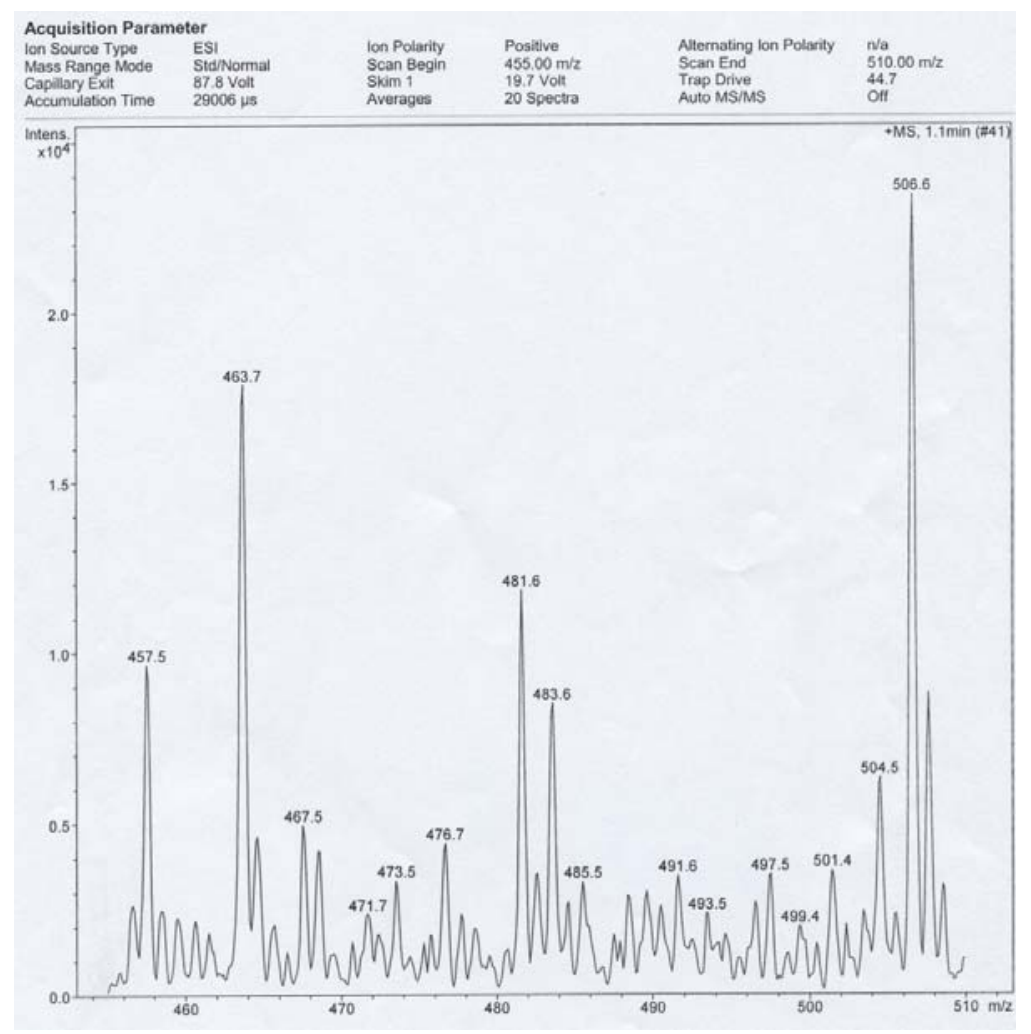




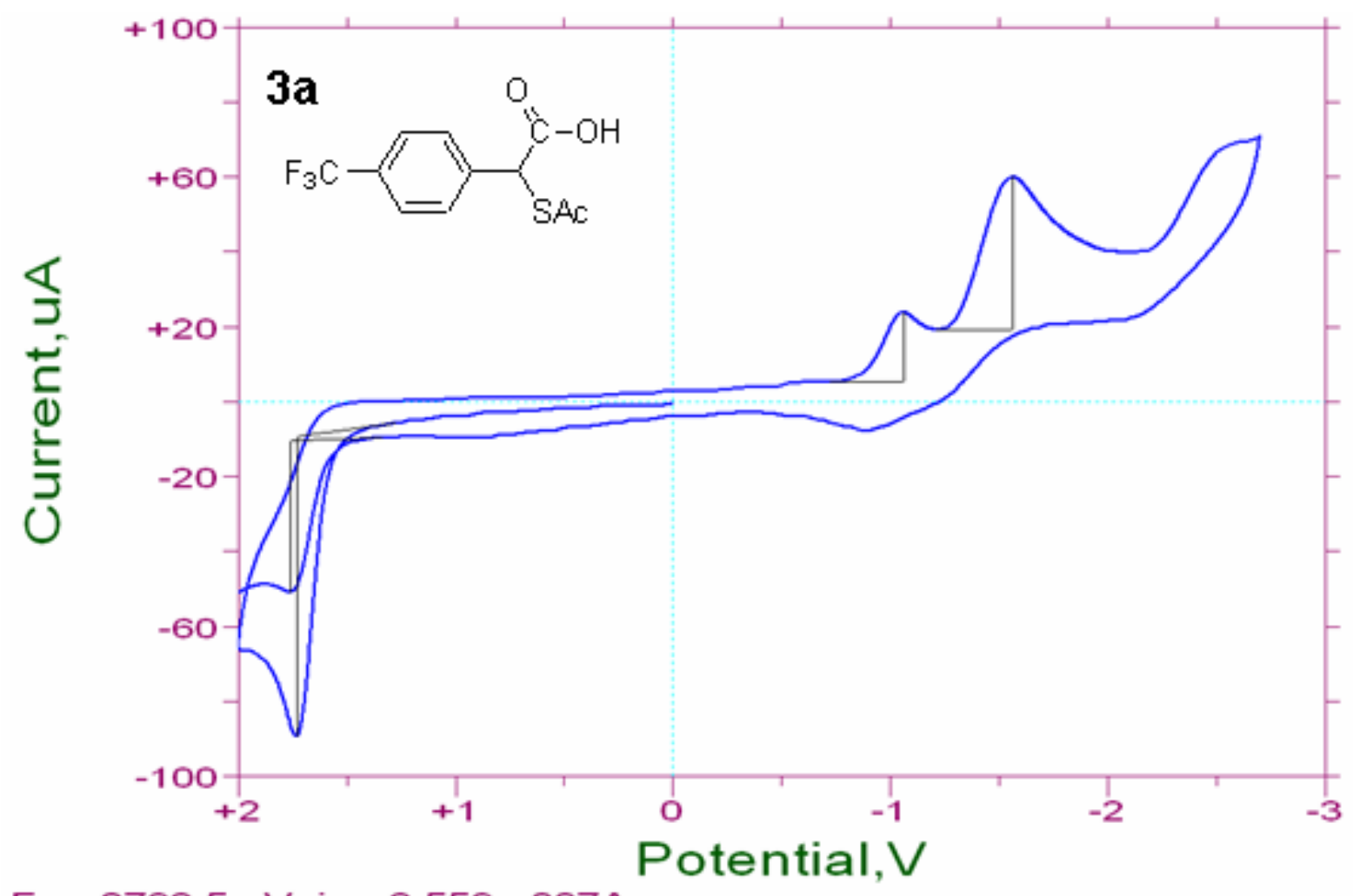

$E=-2793.5 m V i=-3.559 e-007 A$

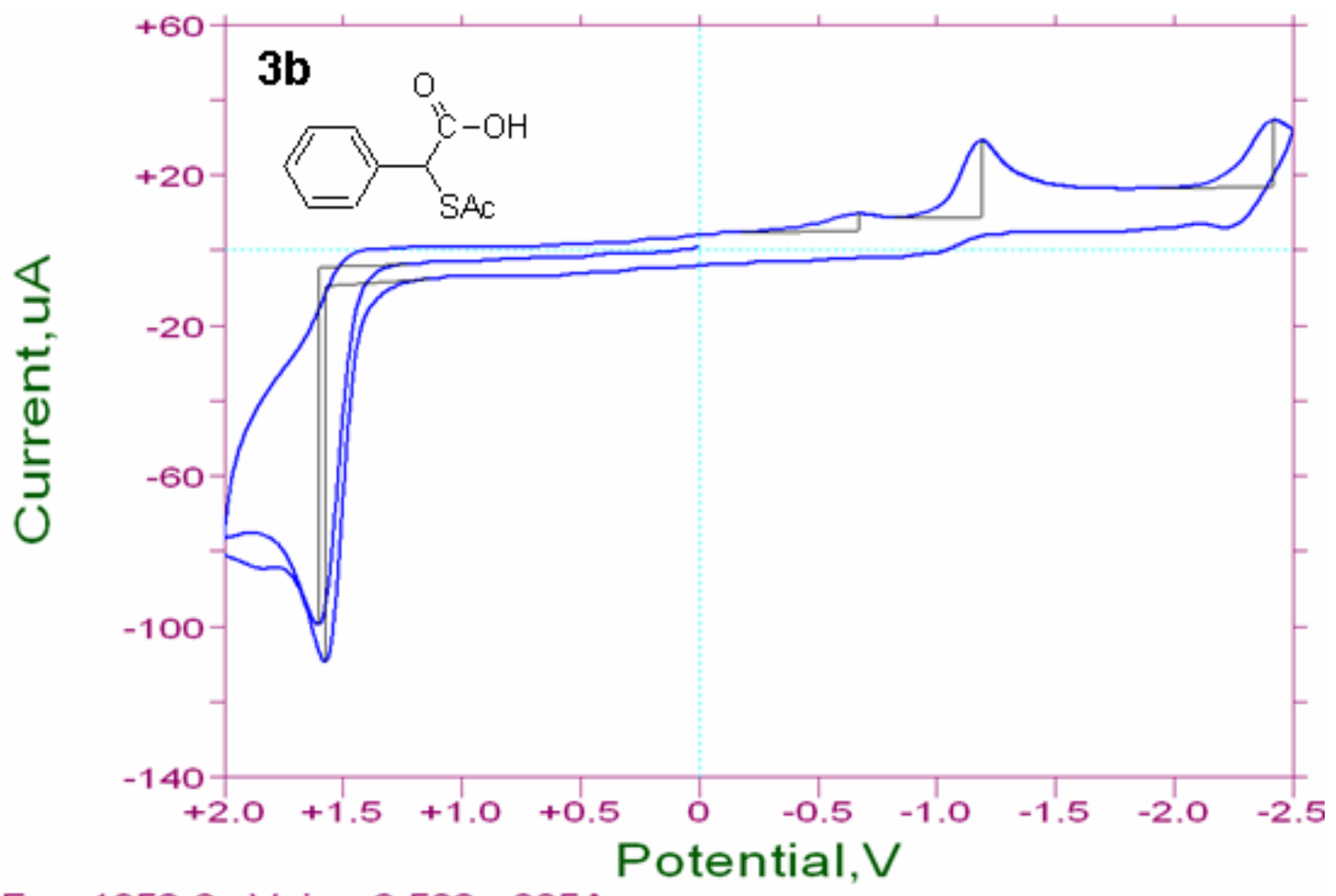

$E=-1052.6 m V i=-3.502 e-005 A$ 


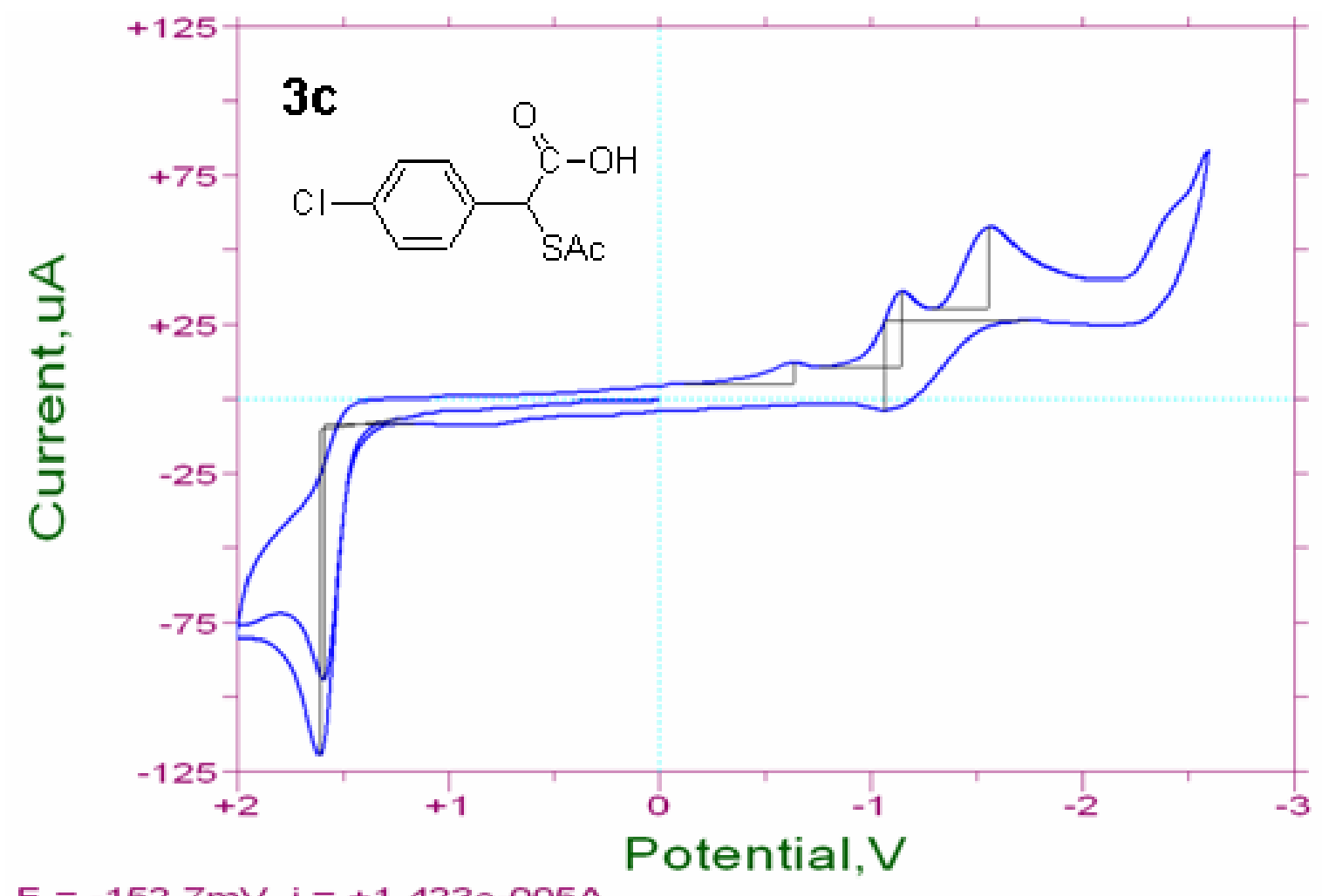

$E=-152.7 m V i=+1.423 e-005 A$

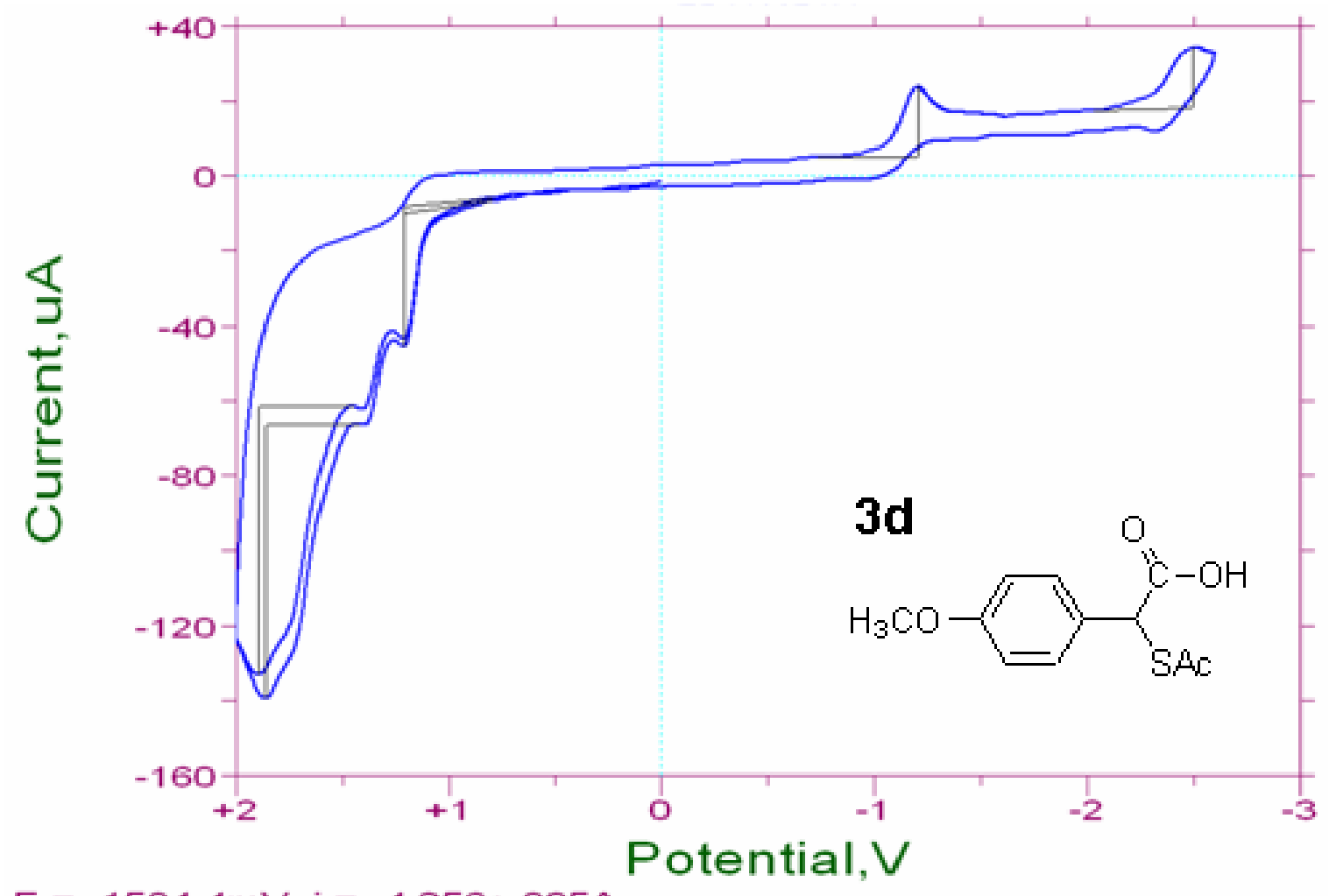

$E=-1504.4 m V i=-4.256 e-005 A$ 
Table S1: Thermodynamic parameters for the ring-opening mechanism of aryl-substituted caged S-nitrosothiols at the PCM/B3LYP/6-311+g**//B3LYP/6_31g*

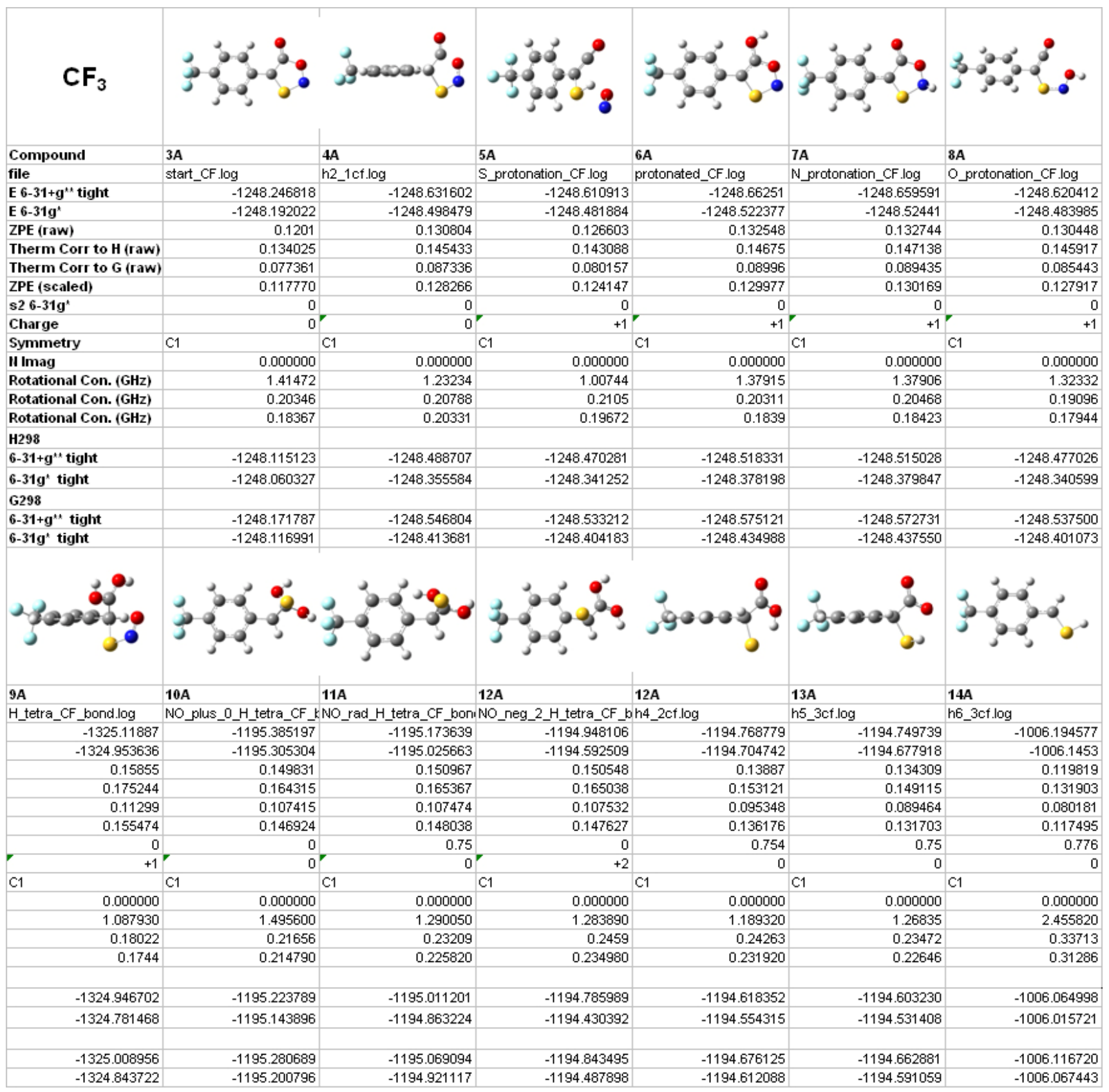




\begin{tabular}{|c|c|c|c|c|c|c|}
\hline & $\int_{0}^{3} a_{0}^{3}$ & 8 & 30 & $\int_{0}^{0} \sigma_{0}^{2} a_{0}^{0}$ & 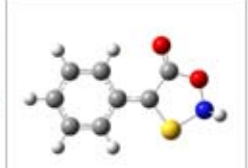 & $j_{0}^{30}$ \\
\hline Compound & 3B & 4B & 5B & 611 & $7 I I$ & 8B \\
\hline file & start_H.log & h2_1 log & S_protonation_H.log & protonated_H.log & N_protonation_H.log & O_protonation_H.log \\
\hline E 6-31+g*k tight & -911.192146 & -911.580999 & -911.558781 & -911.61091 & -911.612067 & -911.568331 \\
\hline E 6-31g & -911.1559873 & -911.4709308 & -911.4544718 & -911.4945859 & -911.4989786 & -911.4556885 \\
\hline ZPE (raw) & 0.11545 & 0.126197 & 0.121985 & 0.127959 & 0.128261 & 0.125797 \\
\hline Therm Corr to H (raw) & 0.125702 & 0.13716 & 0.134834 & 0.138503 & 0.138975 & 0.137626 \\
\hline Therm Corr to G (raw) & 0.079305 & 0.089124 & 0.082207 & 0.091843 & 0.091738 & 0.087251 \\
\hline ZPE (scaled) & 0.113210 & 0.123749 & 0.119618 & 0.125477 & 0.125773 & 0.123357 \\
\hline$s 26-31 g^{t}$ & 0 & 0 & 0 & 0 & 0 & 0 \\
\hline Charge & 0 & 0 & +1 & +1 & +1 & +1 \\
\hline Symmetry & $\mathrm{C} 1$ & $\mathrm{C} 1$ & C1 & $\mathrm{C} 1$ & $\mathrm{C} 1$ & $\mathrm{C} 1$ \\
\hline II Imag & 0.000000 & 0.000000 & 0.000000 & 0.000000 & 0.000000 & 0.000000 \\
\hline Rotational Con. (GHz) & 1.89158 & 1.65681 & 1.26672 & 1.82767 & 1.82631 & 1.74078 \\
\hline Rotational Con. (GHz) & 0.57404 & 0.55951 & 0.5331 & 0.57046 & 0.57583 & 0.50008 \\
\hline Rotational Con. (GHz) & 0.4404 & 0.51895 & 0.49779 & 0.44048 & 0.43867 & 0.42836 \\
\hline \multicolumn{7}{|l|}{ H298 } \\
\hline $6-31+g^{* *}$ tight & -911.068684 & -911.446287 & -911.426314 & -911.474889 & -911.475580 & -911.433145 \\
\hline 6-31g' tight & -911.032525 & -911.336219 & -911.322004 & -911.358565 & -911.362492 & -911.320503 \\
\hline \multicolumn{7}{|l|}{ G298 } \\
\hline $6-31+g^{* t}$ tight & -911.115081 & -911.494323 & -911.478941 & -911.521549 & -911.522817 & -911.483520 \\
\hline 6-31g* tight & -911.078922 & -911.384255 & -911.374631 & -911.405225 & -911.409729 & -911.370878 \\
\hline 8 & ${ }_{3}^{30}$ & (2) & 3 & 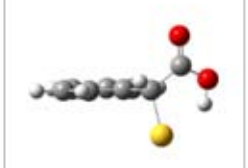 & 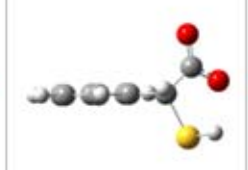 & $\int_{0}^{30} 0_{0}^{2}$ \\
\hline $9 B$ & 10B & 11B & 12B & 12B & 13B & 14B \\
\hline H_tetra_H_bond.log & NO_plus_0_H_tetra_H_bc & CNO_rad_H_tetra_H_bond & NO_neg_2_H_tetra_H_bo & h4_2.log & h5_3.log & h6_3.log \\
\hline-988.065627 & -858.328724 & -858.121887 & -857.910819 & -857.719934 & -857.697331 & -669.136573 \\
\hline-987.9247016 & -858.2677177 & -857.9979583 & -857.5731488 & -857.669622 & -857.51232 & -669.1065855 \\
\hline 0.153896 & 0.145098 & 0.146435 & 0.146131 & 0.134207 & 0.129696 & 0.114892 \\
\hline 0.166971 & 0.155936 & 0.157148 & 0.156932 & 0.1448 & 0.140815 & 0.123396 \\
\hline 0.114513 & 0.109144 & 0.109568 & 0.109802 & 0.097084 & 0.091721 & 0.081655 \\
\hline 0.150910 & 0.142283 & 0.143594 & 0.143296 & 0.131603 & 0.127180 & 0.112663 \\
\hline 0 & 0 & 0.75 & 0 & 0.754 & 0.75 & 0.776 \\
\hline+1 & 0 & 0 & +2 & 0 & 0 & 0 \\
\hline $\mathrm{C} 1$ & $\mathrm{C} 1$ & C1 & C1 & $\mathrm{Cl}$ & C1 & C1 \\
\hline 0.000000 & 0.000000 & 0.000000 & 0.000000 & 0.000000 & 0.000000 & 0.000000 \\
\hline 1.363730 & 2.097620 & 1.714120 & 1.800550 & 1.576080 & 1.707440 & 4.888170 \\
\hline 0.46462 & 0.57091 & 0.6331 & 0.65996 & 0.67992 & 0.64896 & 0.99088 \\
\hline 0.427 & 0.560750 & 0.586330 & 0.602800 & 0.603530 & 0.584050 & 0.82387 \\
\hline-987.901642 & -858.175603 & -857.967580 & -857.756722 & -857.577738 & -857.559032 & -669.015406 \\
\hline-987.760716 & -858.114597 & -857.843651 & -857.419052 & -857.527426 & -857.374021 & -668.985418 \\
\hline-987.954100 & -858.222395 & -858.015160 & -857.803852 & -857.625454 & -857.608126 & -669.057147 \\
\hline-987.813174 & -858.161389 & -857.891231 & -857.466182 & -857.575142 & -857.423115 & -669.027159 \\
\hline
\end{tabular}




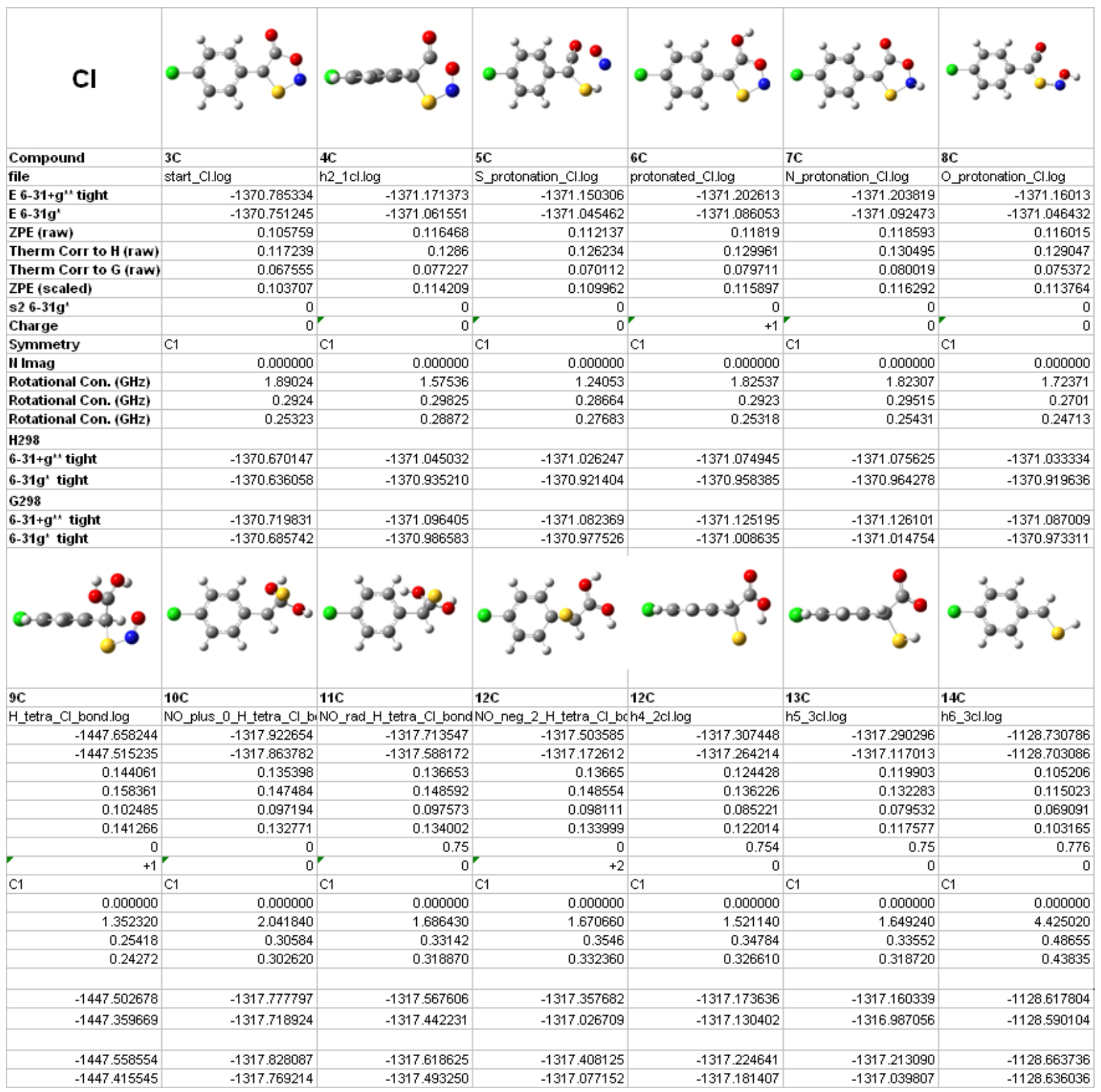




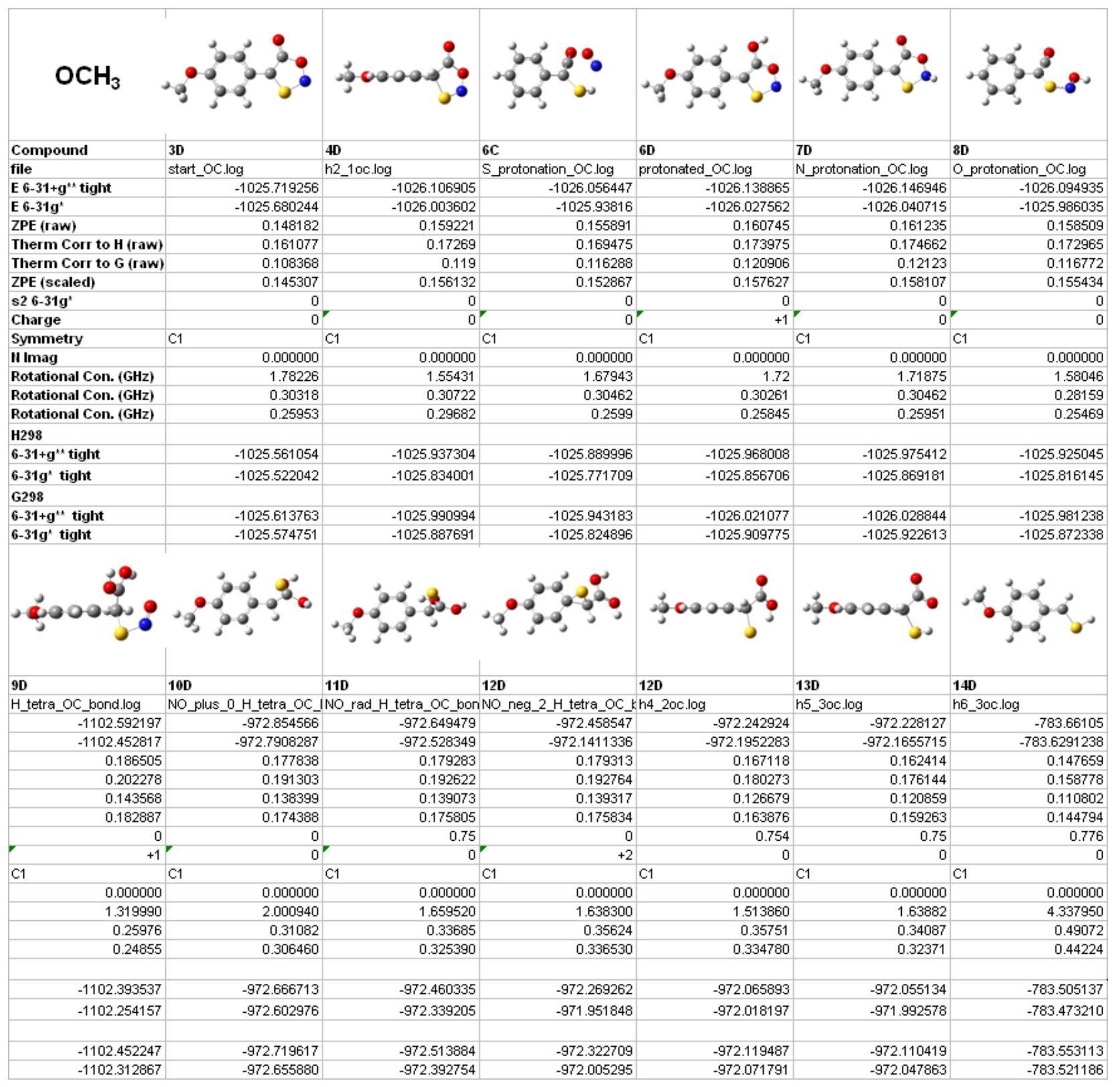

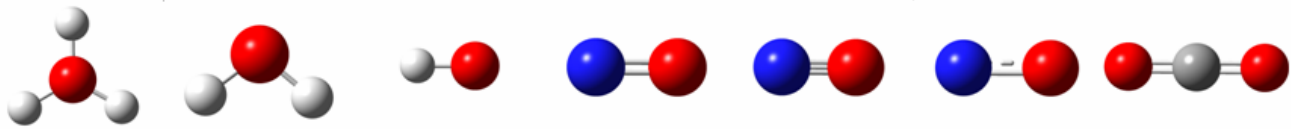

\begin{tabular}{|c|c|c|c|c|c|c|c|}
\hline Compound & H3O & H2O & но- & NO radical & $\mathrm{HO}+$ & 3NO- & $\mathrm{CO} 2$ \\
\hline file & $\mathrm{H} 3 \mathrm{Ob} \cdot \log$ & $\mathrm{H} 2 \mathrm{O} \cdot \log$ & hydroxide.log & NO.log & NO_pos.log & Nitroxyl-triplet.log & $\mathrm{CO} 2 \cdot \log$ \\
\hline E 6-31+g* tight & -76.845424 & -76.444738 & -75.93692 & -129.891647 & -129.6644544 & -130.05632 & -188.58966 \\
\hline E 6-319* & -76.6890842 & -76.40895332 & -75.72077377 & -129.8881562 & -129.52414 & -129.8657549 & -188.5809402 \\
\hline ZPE (raw) & 0.034304 & 0.021168 & 0.007784 & 0.004536 & 0.00565 & 0.003343 & 0.011593 \\
\hline Therm Corr to $\mathrm{H}$ (raw) & 0.038139 & 0.024947 & 0.011089 & 0.007841 & 0.008955 & 0.006653 & 0.015184 \\
\hline Therm Corr to G (raw) & 0.015147 & 0.003501 & -0.008512 & -0.015470 & -0.013556 & -0.017233 & -0.009126 \\
\hline ZPE (scaled) & 0.033639 & 0.020757 & 0.007633 & 0.004448 & 0.005540 & 0.003278 & 0.011368 \\
\hline s2 6-31g* & 0 & 0 & 0 & 0.7525 & 0 & 2.011300 & \\
\hline Symmetry & - & $\mathrm{C} 2 \mathrm{~V}$ & $c^{*} \mathrm{~V}$ & $c^{*} \mathrm{~V}$ & $C * \mathrm{~V}$ & $\mathrm{C} * \mathrm{~V}$ & $D^{*} H$ \\
\hline U Imag & 0.000000 & 0.000000 & 0.000000 & 0.000000 & 0.000000 & 0 & 0.000000 \\
\hline Rotational Con. (GHz) & 323.8423659 & 787.90918 & 0.000000 & 0.000000 & 0.000000 & 0 & 0.000000 \\
\hline Rotational Con. (GHz) & 323.5417689 & 432.30875 & 546.78674 & 50.414284 & 58.801694 & 41.3799784 & 11.5574062 \\
\hline Rotational Con. (GHz) & 186.8424633 & 279.14688 & 546.78674 & 50.414284 & 58.801694 & 41.3799784 & 11.557406 \\
\hline \multicolumn{8}{|l|}{ H298 } \\
\hline $6-31+g^{* \star}$ tight & -76.807950 & -76.420202 & -75.925982 & -129.883894 & -129.655609 & -130.049732 & -188.574701 \\
\hline 6-31g* tight & -76.651611 & -76.384417 & -75.709836 & -129.880403 & -129.515295 & -129.859167 & -188.565981 \\
\hline \multicolumn{8}{|l|}{ G298 } \\
\hline $6-31+g^{* *}$ tight & -76.830942 & -76.441648 & -75.945583 & -129.907205 & -129.678120 & -130.073618 & -188.599011 \\
\hline 6-31g* tight & -76.674603 & -76.405863 & -75.729437 & -129.903714 & -129.537806 & -129.883053 & -188.590291 \\
\hline
\end{tabular}




\section{Complete ref (83)}

(83) Frisch, M. J.; Trucks, G. W.; Schlegel, H. B.; Scuseria, G. E.; Robb, M. A.; Cheeseman, J. R.; Montgomery, J. A., Jr.; T. Vreven; Kudin, K. N.; Burant, J. C.; Millam, J. M.; Iyengar, S. S.; Tomasi, J.; Barone, V.; Mennucci, B.; Cossi, M.; Scalmani, G.; Rega, N.; Petersson, G. A.; Nakatsuji, H.; Hada, M.; Ehara, M.; Toyota, K.; Fukuda, R.; Hasegawa, J.; Ishida, M.; Nakajima, T.; Honda, Y.; Kitao, O.; Nakai, H.; Klene, M.; Li, X.; Knox, J. E.; Hratchian, H. P.; Cross, J. B.; Adamo, C.; Jaramillo, J.; Gomperts, R.; Stratmann, R. E.; Yazyev, O.; Austin, A. J.; Cammi, R.; Pomelli, C.; Ochterski, J. W.; Ayala, P. Y.; Morokuma, K.; Voth, G. A.; Salvador, P.; Dannenberg, J. J.; Zakrzewski, V. G.; Dapprich, S.; Daniels, A. D.; Strain, M. C.; Farkas, O.; Malick, D. K.; Rabuck, A. D.; Raghavachari, K.; Foresman, J. B.; Ortiz, J. V.; Cui, Q.; Baboul, A. G.; Clifford, S.; Cioslowski, J.; Stefanov, B. B.; Liu, G.; Liashenko, A.; Piskorz, P.; Komaromi, I.; Martin, R. L.; Fox, D. J.; Keith, T.; Al-Laham, M. A.; Peng, C. Y.; Nanayakkara, A.; Challacombe, M.; Gill, P. M. W.; Johnson, B.; Chen, W.; Wong, M. W.; Gonzalez, C; Pople, J. A. Gaussian 03, Revision B.04; Gaussian, Inc.: Pittsburgh PA, 2003. 